\title{
A saliency-based bottom-up visual attention model for dynamic scenes analysis
}

\author{
David F. Ramirez-Moreno • Odelia Schwartz • \\ Juan F. Ramirez-Villegas
}

Received: 3 October 2011 / Accepted: 13 December 2012

(C) Springer-Verlag Berlin Heidelberg 2013

\begin{abstract}
This work proposes a model of visual bottomup attention for dynamic scene analysis. Our work adds motion saliency calculations to a neural network model with realistic temporal dynamics [(e.g., building motion salience on top of De Brecht and Saiki Neural Networks 19:14671474, (2006)]. The resulting network elicits strong transient responses to moving objects and reaches stability within a biologically plausible time interval. The responses are statistically different comparing between earlier and later motion neural activity; and between moving and non-moving objects. We demonstrate the network on a number of synthetic and real dynamical movie examples. We show that the model captures the motion saliency asymmetry phenomenon. In addition, the motion salience computation enables sudden-onset moving objects that are less salient in the static scene to rise above others. Finally, we include strong consideration for the neural latencies, the Lyapunov stability, and the neural properties being reproduced by the model.
\end{abstract}

David F. Ramirez-Moreno and Juan F. Ramirez-Villegas contributed equally to the research reported in this work.

D. F. Ramirez-Moreno ( $\varangle)$ · J. F. Ramirez-Villegas

Computational Neuroscience, Department of Physics, Universidad

Autonoma de Occidente, Cali, Colombia

e-mail: dramirez@uao.edu.co

O. Schwartz

Dominick P. Purpura Department of Neuroscience,

Albert Einstein College of Medicine, Yeshiva University,

New York, NY, USA

O. Schwartz

Department of Systems and Computational Biology,

Albert Einstein College of Medicine, Yeshiva University,

New York, NY, USA
Keywords Visual attention - Saliency map · Motion saliency · Neural network · Synaptic depression · Neural latency · Asymmetry phenomenon . Lyapunov stability

\section{Introduction}

Primates' visual cortex is capable of interpreting complex dynamical scenes in clutter. This process is thought to involve attention shifting; i.e., selecting circumscribed regions of visual information to be preferentially processed and by changing the processing focus over the time course. There have been several approaches in the literature for dynamic attention along the ventral and dorsal pathways, including both scene-dependent (bottom-up) and/or task-dependent (top-down) strategies (Itti and Koch 2000; De Brecht and Saiki 2006; Bergen and Julesz 1983; Treisman et al. 1977; Treisman and Gelade 1980); and the interactions between these two processes (Fix et al. 2010; Navalpakkam and Itti 2002, 2005, 2006; Torralba et al. 2006; Walther and Koch 2006; Wolfe et al. 2003).

Many computational models of human visual search have embraced the idea of a saliency map to accomplish preattentive selection. This representation contains the overall neural activity elicited by objects and non-objects, which compete for processing space, and includes salience for primary visual features such as intensity, orientations, colors, and motion (Bollman et al. 1997; Chen et al. 2003; Li 2002; Mundhenk and Itti 2005; Park et al. 2002; Rapantzikos et al. 2007; Reynolds and Heeger 2009; Maunsell and Treue 2006). In some theoretical and experimental studies, salience maps have been attributed to early visual areas [e.g., (Li 1999, 2002; Zhaoping 2006)]. In addition, some electrophysiological and imaging studies have also shown 
neuronal correlates of salience maps in lateral intraparietal cortex (Kusunoki et al. 2000), frontal eye field (Thompson and Bichot 2005), superior colliculus (Shen and Martin 2007) and amygdala (Gonzalez Andino and Grave de Peralta Menendez 2012; Santos et al. 2011; Trapp et al. 2012). Other approaches have considered the overall salience as a consequence of interactions among multiple feature maps, each encoding the saliency of objects for a specific feature (Itti and Koch 2000). Itti and Koch (2000) introduced a bottom-up model, in calculating multiple visual features and linearly combining the feature maps to obtain a representation for describing the overall saliency of the objects on the static image.

In this work, we focus on bottom-up salienceWe particularly consider a dynamical model by De Brecht and Saiki (2006). The model's primary visual features are a simplified version of Itti and Koch (2000), but their (static image) model is extended to incorporate a neural network that captures temporal dynamics. Their model incorporates at least three aspects: first, their model includes realistic dynamics based on data from electrophysiology experiments. Second, the neural activity normalization during saliency processing avoids exaggeration of the differences among the calculated dimensions (from feature maps to the final saliency map). Finally, over-competition within the maps is avoided by synaptic depression, in addition to a linear gain function with a threshold of zero. Synaptic depression is the temporary decrease in synaptic efficacy between a highly active presynaptic neuron and the postsynaptic neuron; provided that as it uses up the synaptic resources faster than they can be recovered, the strength of the synaptic connections weakens (Abbott et al. 1997; Tsodyks et al. 1998). This model was shown to be useful for saccadic eye movements modeling during free vision tasks and for capturing sudden-onset stimuli, provided that the moving object is itself salient.

The present work develops a saliency-based visual attention model for dynamical scene analysis. Our approach expands the results obtained by Itti and Koch (2000) and De Brecht and Saiki (2006), by incorporating a motion saliency computation (not present even in the dynamical approach of De Brecht and Saiki). Our bottom-up model integrates the motion saliency computation over time. This avoids a problem faced by the dynamical model of De Brecht and Saiki (2006) when it is applied to moving targets or to certain sudden-onset ones; i.e., in their model, the transient response of moving (or sudden-onset) non-salient stimuli cannot be forced to rise above the other objects by the network dynamics itself. Therefore, we propose that adding a motion salience computation enables to capture salience of a sudden-onset target which is not deemed salient in the absence of motion (i.e., is not salient in the static case, in comparison to other objects). In addition, our model keeps the synaptic depression mechanism for motion saliency computations in order to avoid over competition and undesired effects when the moving target is other than the most salient one.

The final saliency map is implemented as an array of neural populations with dynamic synapses and with dynamic motion detection. We used Naka-Rushton functions in mean field equations with dynamic synapses in order to describe the neurons' spike rates. Under this representation, the model dynamics itself causes the target to rise above the distractors even when competition occurs among all neurons in the map.

Neurons in primary visual cortex perform the first stage of cortical motion processing, which continues along the dorsal pathway. Spatiotemporal filters are commonly used to model the selectivity of V1 neurons for speed and direction of local motion (Schrater et al. 2000; Borst 2000; Chen 2005; Ibbotson 2001). Here, we adopt this approach and use a simple implementation of motion detection. Experimental evidence shows that sudden-onset stimuli or moving stimuli increase their priority during the transient response of the neurons and this priority decays if the given stimulus stops moving, so that the neural assembly reaches its steady state response (Yantis 1993). This suggests that motion is, as expected, a primary visual feature dynamically computed by the visual system given the time response of the neural assembly involved in bottom-up saliency processing (Itti and Koch 2000; De Brecht and Saiki 2006). Previous work also indicates that motion detection requires a set of non-linear interactions between locations and instances in time (Meso and Zanker 2009; Sejnowski et al. 1988; Shormaker et al. 2001). As the models of Itti et al. (1998) and De Brecht and Saiki (2006) have shown, the motion salience computation cannot be achieved without the presence of motion detectors. In our model, motion detectors are implemented and embedded into the dynamical equations for the computation of motion salience.

We demonstrate our dynamical model with motion salience examples for both real and synthetic dynamic scenes. In Sect. 2.3 we introduce the dynamical model. In Sect. 3.1.2, we discuss the saliency processing latencies and show that the model reproduces the expected transitory responses under different experimental frames. We also address motion saliency asymmetries and illustrate motion saliency for dynamic scenes. We make a comparison between the models with and without motion detectors, showing that the motion detectors are necessary to capture the salience of sudden-moving objects. In Sect. 3.2, we consider in detail the Lyapunov stability in the model's dynamics and in Sect. 4 we explore possible integrations to top-down mechanisms. 


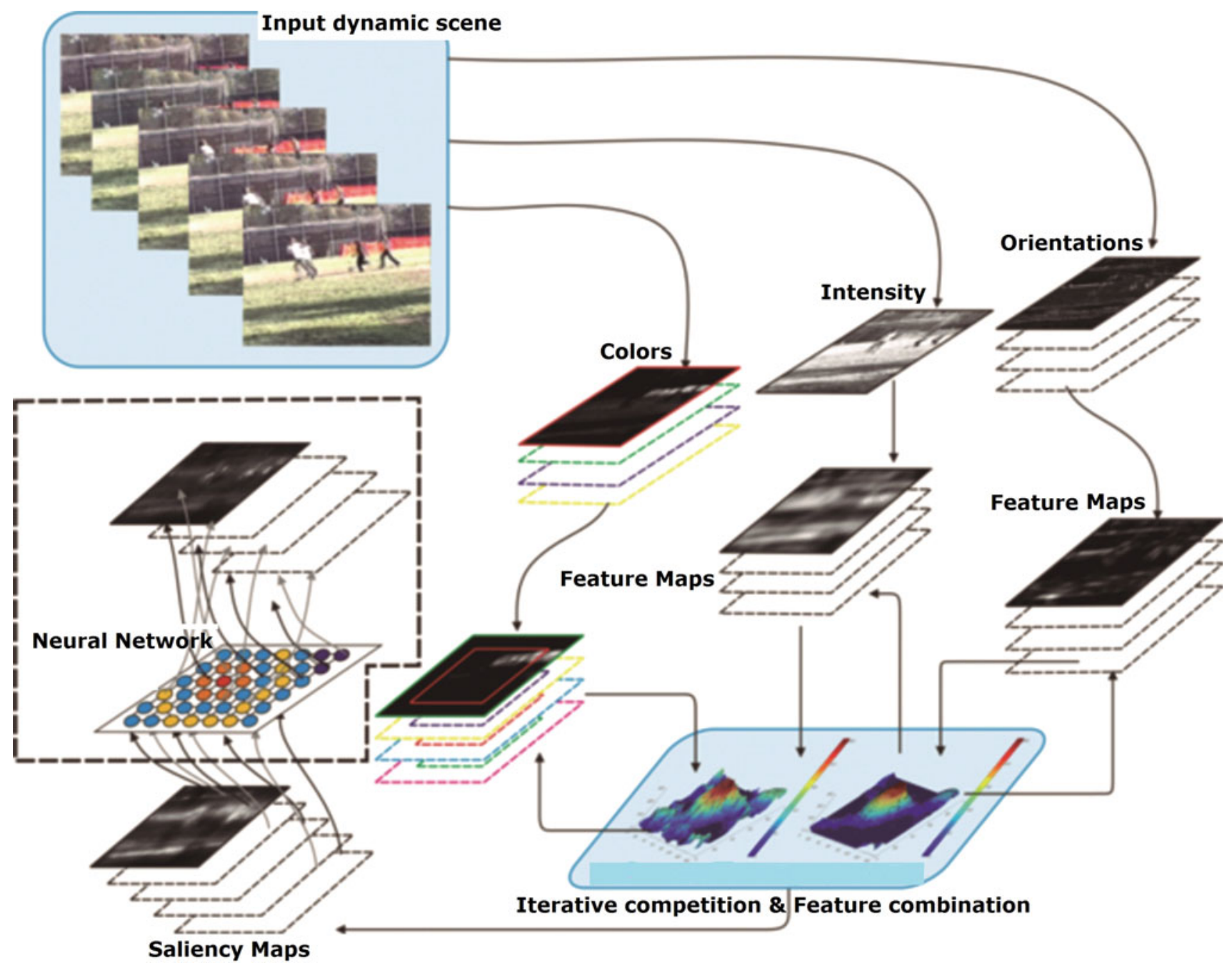

Fig. 1 Flow diagram for the bottom-up visual processing model proposed in this work. The novel part of the model is surrounded by the dashed line

\section{Model}

\subsection{Model's overview}

The model proposed in this work is based on the Itti and Koch (2000) and De Brecht and Saiki (2006) saliency maps models. This model computes saliency using the three features studied by Itti and Koch: intensity, orientation, and color. These features are organized into 54 maps ( 6 for intensity, 24 for orientation, and 24 for color; a detailed explanation of this is given further). These maps are combined using across-scale sums in order to obtain the conspicuity maps, which provide input for a unique saliency map implemented as an array of neural populations (Fig. 1). These populations are interconnected by an elliptical Gaussian-shape function, which maintains the schemes of collaboration and competition between local and distant populations, respectively.
Motion detection in our model is accomplished by subtracting consecutive neural inputs into the dynamical equations. We found the results of this procedure highly similar to standard motion detectors (over the main peak of the transient response of the system). Given the systems' dynamics, a population's Gaussian-shaped connections induce smoothing and therefore noise reduction. De Brecht and Saiki (2006) computed visual salience using a network with dynamic synapses and responses in time. Although their model could be suitable to the dynamic scenes analysis, their approach does not integrate the computation of motion salience.

Under the setup proposed in this work, the transient response of the non-linear system exhibits an overshoot (or a sequence of maximum peaks) when the stimulus suddenly moves from a place to another (or makes a sequence of movements) over different scene frames; then, when the stimulus stops moving the system reaches its steady state response, i.e., the moving stimulus priority decays. 


\subsection{Primary visual features extraction}

As stated previously, the feature extraction procedure expands over three main well-studied feature maps: intensity, orientation, and color maps. The center-surround differences are implemented using fine and coarse scales of the Laplacian pyramid (Burt et al. (1983)) for each feature: the receptive center corresponds to a pixel at resolution level $c \in\{2,3,4\}$ and the surround is the corresponding pixel at level $s=c+\delta$, with $\delta \in\{3,4\}$.

Contrast intensity is extracted by standard band-pass filtering to calculate center surround differences between the established resolution levels:

$I(c, s)=|I(c) \Theta I(s)|$,

where $I(c)$ is the center intensity signal, $I(s)$ is surround intensity signal and the symbol " $\Theta$ " is termed across-scale subtraction, i.e., standard subtraction of images of different resolutions by interpolation to the finer scale.

Orientation is extracted using standard Gabor pyramids $O(\theta, \sigma)$, where $\theta \in\left\{0^{\circ}, 45^{\circ}, 90^{\circ}, 135^{\circ}\right\}$ (Greenspan et al. 1994). Thereby, orientation contrast is defined as:

$O(c, s, \theta)=|O(c, \theta) \Theta O(s, \theta)|$,

where $O(c, \theta)$ y $O(s, \theta)$ are the center and surround orientation signals, respectively.

We established four different color maps according to four broadly tuned colors, namely $R G(c, s), B Y(c, s), R C(c, s)$, and $G M(c, s)$ to account for red/green, blue/yellow, red /cyan, and green/magenta opponency (T'so and Gilbert 1988; Conway 2001, 2009; Engel et al. 1997). Conway (2001) established the existence of certain groups of cells sensitive to another type of contrast color. According to his experiments, for red-on (L+) or yellow-on (S-) stimuli, the center of the cell is excited and at the same time is chromatically opponent to green-on $(\mathrm{M}+)$ and blue-on $(\mathrm{S}+)$ stimuli (in the surround region). This phenomenon also occurs for green-on center sensitive cells. Both the antagonism and the alignment of $\mathrm{M}$ and $\mathrm{S}$ cell stimuli are suggestive of the existence of red-cyan and green-magenta cells. Ramirez-Villegas and RamirezMoreno (2012) recently demonstrated that a color model driven by red-cyan and green-magenta cells enhances the whole visual content of natural scenes, i.e., a fully enhanced output nonlinearity that predicts human eye fixations with higher accuracy:

$$
\begin{aligned}
& R=r-\frac{(g+b)}{2}, \\
& G=g-\frac{(r+b)}{2}, \\
& B=b-\frac{(g+r)}{2},
\end{aligned}
$$

$Y=\frac{(r+g)}{2}-\frac{|r-g|}{2}-b$,

$R G(c, s)=|(R(c)-G(c)) \Theta(G(s)-R(s))|$,

From Eq. (7), we derive red-cyan double opponent cells as follows:

$$
\begin{aligned}
R C(c, s)= & \mid(R(c)+Y(c)-G(c)-B(c)) \Theta(G(s) \\
& +B(s)-R(s)-Y(s)) \mid, \\
B Y(c, s)= & |(B(c)-Y(c)) \Theta(Y(s)-B(s))|,
\end{aligned}
$$

Finally, green-magenta opponency is calculated as:

$$
\begin{aligned}
G M(c, s)= & \mid(G(c)+Y(c)-R(c)-B(c)) \Theta(R(s) \\
& +B(s)-G(s)-Y(s)) \mid
\end{aligned}
$$

For all the former equations, $R, G, B$, and $Y$ are the red, green, blue, and yellow broadly tuned color channels, respectively; $R(c), G(c), B(c)$, and $Y(c)$ are the center color signals and $R(s), G(s), B(s)$, and $Y(s)$ are the surround color signals.

\subsection{Dynamical model: mathematics and relation to neurobiology}

Once the 54 feature maps are created, they are combined using across-scale sums in order to obtain the conspicuity maps (one for each feature) and then a first representation of the total saliency of the objects in the scene is created with equal contribution of these conspicuity maps (Itti and Koch (2000)).

The diagram in Fig. 2a,b illustrates the interactions and the connectivity among the units of our neural network model with motion dynamics. Note that the units $A$ receive the visual information about all the inputs coming from the dynamic scene, either a target or distractor; the distinction among them is a consequence of the dynamic behavior of the network, in which all the units representing the stimuli compete over time [see Fig. 2a,b; Eqs. (11-14)]. Note that the targets and distractors are not input parameters into the model; they are determined directly by the neural network based on competition.

The final saliency map is implemented as an array of neural populations with dynamic synapses and with dynamic motion detection. Rather than implementing each neuron and their synapses, we used Naka-Rushton functions in mean field equations with dynamic synapses in order to describe the neurons' spike rates temporal variations (they are derived from the Wilson (2004) winner-take-all network approaches). The Naka-Rushton function $f(x)$ is defined by the next two mathematical relations where $c$ is the maximum reachable value or the saturation value and $\sigma$ is the semisaturation constant, i.e., the value of $x$ for which $f(x)$ is half of $c$. 


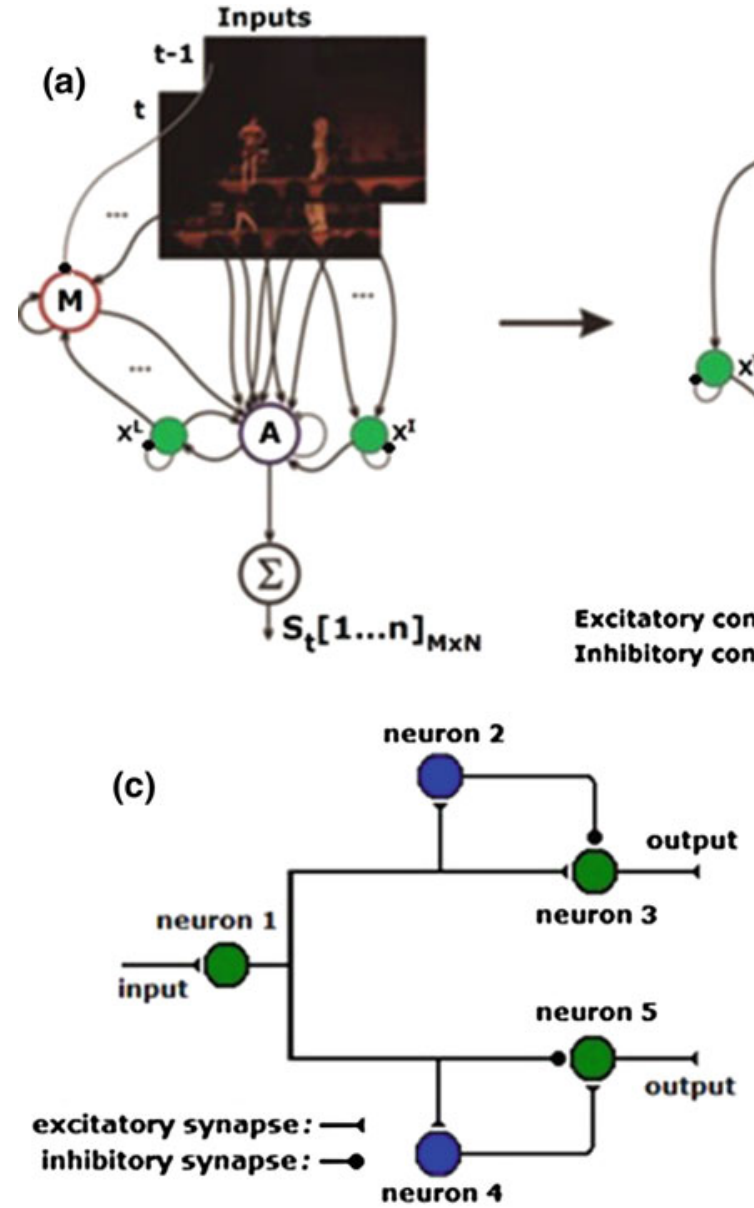

Fig. 2 Neural network diagrams: a general model diagram illustrating the interactions among all neurons (blue, $A$ ); motion saliency (red, $M$ ), and the synaptic depression mechanism (green, $x^{L}$ and $x^{I}$ ). b Specific diagram illustrating the interactions among the units representing targets and distractors (blue, $T$ and $D$ ) once they have been coded by the network. Motion saliency units are in red $\left(M^{T}\right.$ and $\left.M^{D}\right)$ and those representing the synaptic depression mechanism are in green

$$
\begin{aligned}
& f(x)=\frac{C \cdot(x)_{+}^{2}}{\sigma^{2}+(x)_{+}^{2},} \\
& (x)_{+}=\left\{\begin{array}{l}
x, \text { if } x>0 \\
0, \text { if } x \leq 0
\end{array},\right.
\end{aligned}
$$

Theoretically, if there were no center-surround interactions among neural populations and no synaptic depression for the input and the neural population's activity, there would be one single neuron winning the competition for all cases; therefore, the winner-take-all (WTA) neural network's dynamics would choose a unique target if and only if this target corresponds to the maximum input. In other words, the network would exaggerate the differences between the neurons' activities leading to undesired over-competition.

The Eqs. (13-16) are built following the model of Wilson and Cowan (1973), and explain the dynamics of the
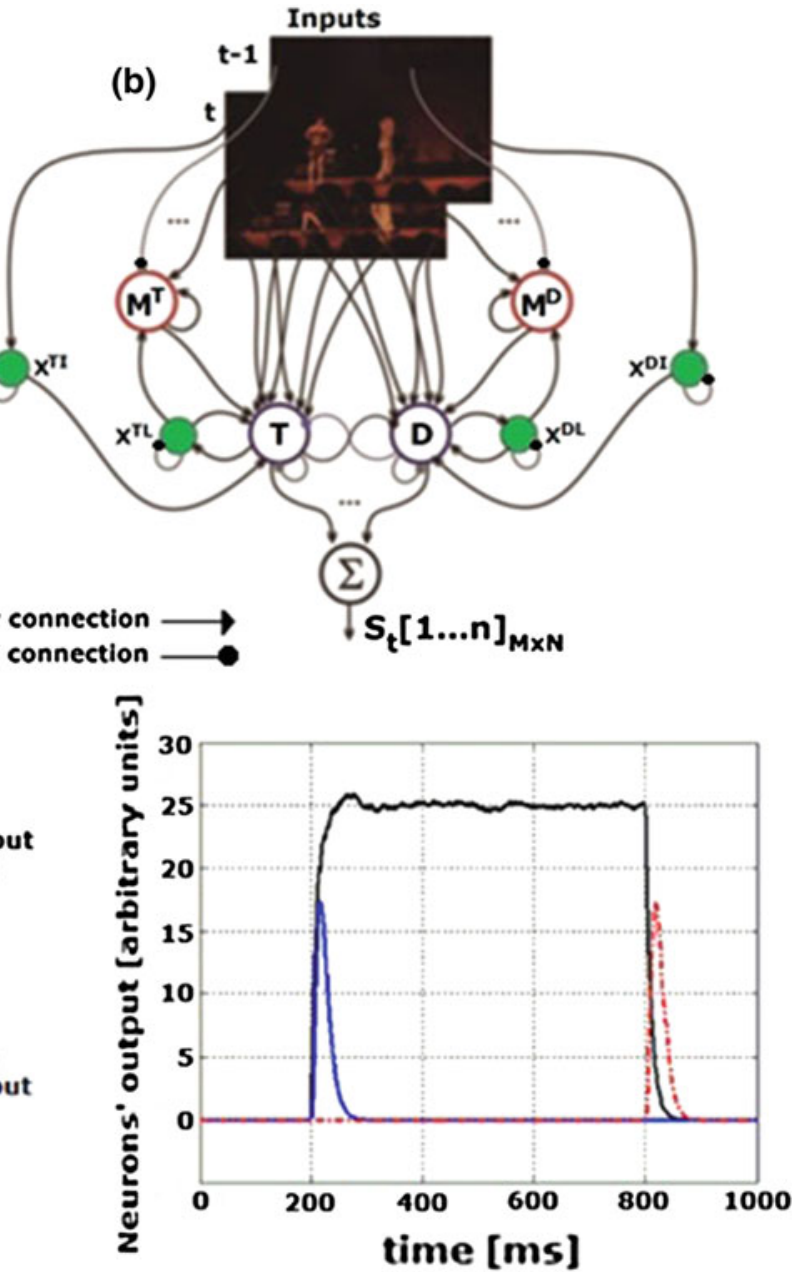

$\left(x^{T L}, x^{D L}, x^{T I}\right.$ and $\left.x^{D I}\right)$; the final unit of the model is responsible for integrating the system over time. The outputs are a measure of the firing rates of the units representing the saliency of the overall dynamical scene, which is composed by frames of size $M_{\mathrm{x}} N$. c Left simple synaptic circuit for motion detection via computation of temporal changes. Right neuron $3^{\prime}$ s and neuron $5^{\prime}$ s response (blue and red, respectively) for a step input (black) coming from neuron 1

neural network model proposed in this work. It should be noted that the diagrams in Fig. 2 illustrate the interactions among the processing units in the Naka-Rushton terms [second terms in the right side of (13) and (14)], and in the synaptic depression terms [second terms in (15) and (16)]. In this way, the positive terms in the Naka-Rushton function are represented by an excitatory connection (marked with arrows); conversely, the negative terms in the Naka-Rushton function are represented by an inhibitory connection (marked with dots) in Fig. 2. Note that this representation reflects perfectly the general dynamics of the system: the input movie frames are shown to the network and they excite every processing unit $A$, which competes in parallel (every unit inhibits each other); the synaptic depression terms and the motion detection term act on the $A$ processing units in order to directly raise or depress the activity of these units, according to the 
activity elicited by synaptic depression and motion detection dynamics. Finally, the self-inhibitory connection of the synaptic depression units guarantees that the neural activity of the most active $A$ units decreases and that the activity of the less active $A$ units increases. Accordingly, the equations for the network dynamics are as follow:

$$
\begin{aligned}
& \tau_{1} \frac{\mathrm{d} A_{i}}{\mathrm{~d} t}=-A_{i}+f\left(E_{i k}-r \sum_{j=1}^{N-1} A_{j}+U_{\mathrm{SE}}^{L}\right. \\
& \left.\times\left(\sum_{j} w_{i j}^{\mathrm{L}} E_{j k}\right) x_{i}^{\mathrm{I}}+x_{i}^{\mathrm{L}} A_{i}+M_{i}\right), \\
& \tau_{1} \frac{\mathrm{d} M_{i}}{\mathrm{~d} t}=-M_{i}+f\left(\left(E_{i k}-E_{i(k-1)}\right)+U_{\mathrm{SE}}^{M}\right. \\
& \left.\times\left(\sum_{j} w_{i j}^{L}\left(E_{j k}-E_{j(k-1)}\right)\right)+B x_{i}^{L}\right), \\
& \frac{\mathrm{d} x_{i}^{L}}{\mathrm{~d} t}=-x_{i}^{L} U_{\mathrm{SE}}^{L} A_{i}+\frac{1-x_{i}^{L}}{\tau_{2}}, \\
& \frac{\mathrm{d} x_{i}^{I}}{\mathrm{~d} t}=-x_{i}^{I} U_{\mathrm{SE}}^{I} E_{i k}+\frac{1-x_{i}^{I}}{\tau_{2}},
\end{aligned}
$$

with:

$C=40.0 ; \quad r=3.0 ; \quad \sigma=120.0 ; \quad \tau_{1}=20.0 ;$

$\tau_{2}=10.0 ; \quad U_{\mathrm{SE}}^{\mathrm{M}}=U_{\mathrm{SE}}^{\mathrm{L}}=U_{\mathrm{SE}}^{\mathrm{I}}=1.2 ; \quad B=10.0$

where $A_{i}$ is the spike rate of any neuron receiving an input about the target or distractor, the constant $r$ describes the feedback inhibition strength (neurons' competition); similarly, $M_{i}$ describe the spike rate of any neuron receiving an input about a moving target or an input about a moving distractor; $x_{i}^{L}$ and $x_{i}^{I}$ are the variables representing the synaptic depression between laterally connected populations and afferent populations (De Brecht and Saiki 2006), respectively. As illustrated by the equations of the network, the neuron's net potential at a given time (the squared term in the Naka-Rushton function) is built from the collaborative and competitive interactions between the activities of the neurons representing the saliency map, the synaptic depression mechanism and the dynamical motion detectors. Furthermore, $E_{i k}$ (i.e., the input $E_{i}$ at the frame $k$ ) is the activity of any neuron providing input to the network from the former layer. The lateral connection weights among the neural populations in the saliency map $w_{i j}^{L}$ are determined by an elliptical differenceof-Gaussians function:

$$
\begin{array}{r}
w_{i j}^{L}=\frac{w_{\mathrm{E}}}{2 \pi \sigma_{\mathrm{E} 1}^{2} \sigma_{\mathrm{E} 2}^{2}} \exp \left[-\left(\frac{x^{2}}{2 \sigma_{\mathrm{E} 1}^{2}}+\frac{y^{2}}{2 \sigma_{\mathrm{E} 2}^{2}}\right)\right]-\frac{w_{I}}{2 \pi \sigma_{I 1}^{2} \sigma_{I 2}^{2}} \\
\exp \left[-\left(\frac{x^{2}}{2 \sigma_{I 1}^{2}}+\frac{y^{2}}{2 \sigma_{I 2}^{2}}\right)\right],
\end{array}
$$

where $w_{\mathrm{E}}=5.0 ; \quad w_{I}=250.0 ; \quad \sigma_{\mathrm{E} 1}=0.05^{*} X_{T} ; \quad \sigma_{\mathrm{E} 2}=$ $0.05^{*} Y_{T} \sigma_{I 1}=0.75^{*} X_{T} ; \quad \sigma_{I 2}=0.75^{*} Y_{T}$, where $X_{T}$ and $Y_{T}$ are the number of rows and columns of the saliency map, respectively.

The constants of this function define the form of the Gaussian kernels according to the distances $x$ and $y$. This scheme guarantees center-surround competitive interactions between the populations in the saliency map and enhances the difference between the activity of the neural population representing the target and the activity of those representing the distractors without generating undesired over-competition between populations.

In addition, constants $C, \tau, \sigma$ and $U_{\mathrm{SE}}^{L}$ are adjustable parameters of the system, where $C$ is the maximum spike rate of the system, the time constant $\tau_{n}$ controls the frequency at which $A_{i}, M_{i}, x_{i}^{L}$ and $x_{i}^{I}$ reach their maximum value (in milliseconds), $\sigma$ is the system's semi-saturation constant and $U_{\mathrm{SE}}^{L}$ and $U_{\mathrm{SE}}^{I}$ defines the synaptic efficiency between intraand inter-layer neural connections.

The parameters of this model $\left(C, \tau, \sigma\right.$ and $\left.U_{\mathrm{SE}}^{L}\right)$ were adjusted according to experimental evidence available in the literature (De Brecht and Saiki 2006; Tsodyks et al. 1998; Wilson 2004). In addition, we are introducing parameter $B$, defined as the strength of synaptic depression for motion saliency; it establishes the overall sensitivity of the system to temporal changes, i.e., the magnitude of the overshoot elicited when a stimulus appears or moves. This parameter is critical for over-competitive behavior too, as the overall activity of the distractors weakens remarkably if this parameter lowers. As synaptic depression relative weight increases, it also increases the activity of non-winning neurons and this solves the problem commonly referred to as exaggeration of the differences between the target and distractors (De Brecht and Saiki 2006). We noticed important differences in the populations' activity when we varied this weight by $30 \%$ (amongst the various experiments carried out). On the other hand, the Naka-Rushton parameters (especially $\sigma$ ) of the motion saliency equations, were chosen on the basis of counterbalancing the effect of saturation often presented by Naka-Rushton neurons. That is, if the semisaturation constant is relatively short (with respect to the range of values of the squared term of the equation), then, regardless of their input, nearly all the neurons would have quite the same output, and this is definitely an undesired effect for the overall saliency computation. Thus, it is desirable to have a semi-saturation constant that permits conserving the main features of the input space. In our model, 120 was chosen, given the fact that the values for the squared Naka-Rushton terms were fully distributed in the interval $\left[0,10^{4}\right]$.

We are taking most of the cited parameters from Itti et al. (1998), De Brecht and Saiki (2006), and Wilson (2004), and adding a new parameter related to the motion detection 
term. Therefore, we are adopting their heuristics while introducing a novel saliency-based mechanism for motion detection.

According to our experiments, all these values give a robust response to different stimuli (natural or synthetic); additionally, all the other parameters can be varied by approximately $50 \%$ before large changes in the neural populations responses occur. For the dynamical motion detection, we have taken similar parameters to those considered for the equations describing the competitive dynamics between neural populations, i.e., Eq. (13). Time constants (often referred to as synaptic recovery constants) were taken from the Wilson (2004) competitive models which produce response latencies similar to those of humans (Wilson 2004)

As aforementioned, the competition occurs among every neuron in the map and the network's dynamics produces the activity of the neurons representing the target to rise above the activity of those representing the distractors. Evidently, the response of the system varies according to what is being encoded by one given neuron (see Fig. 3b, for reference). To illustrate this fact clearly, when the input is a target, Eq. (13) can be re-written as:

$$
\begin{aligned}
\tau_{1} \frac{\mathrm{d} T_{i}}{\mathrm{~d} t}=-T_{i}+f & \\
\times & \left(E_{i k}^{T}-r \sum_{j=1}^{N^{\prime}} D_{j}-r \sum_{j=1}^{n-1} T_{j}+U_{\mathrm{SE}}^{L}\left(\sum_{j} w_{i j}^{L} E_{j k}^{T}\right)\right. \\
& \left.x_{i}^{T I}+x_{i}^{T L} T_{i}+M_{i}^{T}\right),
\end{aligned}
$$

where $E_{i k}^{T}$ is the target input $(i, k), T_{i}$ is the neural activity elicited by the target $i$ from a total of $n$ targets; $D_{j}$ is the neural activity elicited by a distractor $j$ in a collection of $N^{\prime}$ distractors, and:

$$
\begin{aligned}
& \tau_{1} \frac{\mathrm{d} M_{i}^{T}}{\mathrm{~d} t}=-M_{i}^{T}+f\left(\left(E_{i k}^{T}-E_{i(k-1)}^{T}\right)+U_{\mathrm{SE}}^{M}\right. \\
& \times\left.\left(\sum_{j} w_{i j}^{L}\left(E_{i k}^{T}-E_{i(k-1)}^{T}\right)\right)+B x_{i}^{T L}\right), \\
& \frac{\mathrm{d} x_{i}^{T L}}{\mathrm{~d} t}=-x_{i}^{T L} U_{\mathrm{SE}}^{L} T_{i}+\frac{1-x_{i}^{T L}}{\tau_{2}} \\
& \frac{\mathrm{d} x_{i}^{T I}}{\mathrm{~d} t}=-x_{i}^{T I} U_{\mathrm{SE}}^{I} E_{i k}^{T}+\frac{1-x_{i}^{T I}}{\tau_{2}},
\end{aligned}
$$

where $x_{i}^{T L}$ and $x_{i}^{T I}$ are the variables representing the synaptic depression mechanism for such particular target.

Similarly, when the input is a distractor, (13) can be rewritten as:

$$
\begin{aligned}
\tau_{1} \frac{\mathrm{d} D_{i}}{\mathrm{~d} t}= & -D_{i}+f\left(E_{i k}^{D}-r \sum_{j=1}^{N^{\prime}-1} D_{j}-r \sum_{j=1}^{n} T_{j}+U_{\mathrm{SE}}^{L}\right. \\
& \left.\times\left(\sum_{j} w_{i j}^{L} E_{i k}^{D}\right) x_{i}^{D I}+x_{i}^{D L} D_{i}+M_{i}^{D}\right),
\end{aligned}
$$

where $E_{i k}^{D}$ is the distractor input $(i, k), D_{i}$ is the neural activity elicited by the distractor $i$ from a total of $N^{\prime}$ targets; $T_{j}$ is the neural activity elicited by the target $j$ in a collection of $n$ targets, and:

$$
\begin{aligned}
\tau_{1} \frac{\mathrm{d} M_{i}^{D}}{\mathrm{~d} t}=-M_{i}^{D}+f\left(\left(E_{i k}^{D}-E_{i(k-1)}^{D}\right)+U_{\mathrm{SE}}^{M}\right. \\
\left.\times\left(\sum_{j} w_{i j}^{L}\left(E_{i k}^{D}-E_{i(k-1)}^{D}\right)\right)+B x_{i}^{D L}\right), \\
\frac{\mathrm{d} x_{i}^{D L}}{\mathrm{~d} t}=-x_{i}^{D L} U_{\mathrm{SE}}^{L} D_{i}+\frac{1-x_{i}^{D L}}{\tau_{2}}, \\
\frac{\mathrm{d} x_{i}^{D I}}{\mathrm{~d} t}=-x_{i}^{D I} U_{\mathrm{SE}}^{I} E_{i k}^{D}+\frac{1-x_{i}^{D I}}{\tau_{2}},
\end{aligned}
$$

where $x_{i}^{D L}$ and $x_{i}^{D I}$ are the variables representing the synaptic depression mechanism for such particular distractor. Please note that for all the cases $E_{i k}^{T}>E_{i k}^{D}$.

Next, we explain how a neural circuit could perform the subtraction shown in the right side of Eqs. (14), (19), and (23). Figure 2c, shows a synaptic circuit for projecting into the motion detectors $M_{i}$. Neurons 2 and 4 constitute a simple mechanism for short term memory, relaying an inhibitory and an excitatory delayed signal, respectively, from neuron 1 to neurons 3 and 5 , and giving them the possibility of computing the input temporal change at neuron 1 . This simple mechanism allows for motion detection since neuron 3 and 5 can signal a change coming from reflected light intensity, for instance, falling onto neuron 1's receptive field from a particular space location. This circuit is not shown in Fig. 2a,b and should be placed between the input frames and $M_{i}$.

In addition, De Brecht and Saiki (2006) implemented their model using a linear function for the second term of the mean field equation. However, we preferred to use the NakaRushton function, as a linear function can be approximated using Naka-Rushton parameters for a range of values. Moreover, biological evidence establishes that cell potentials are more suitable to logistic function profiles (Wilson (2004)).

\section{Results}

Our approach fully reproduces the results obtained by Itti and Koch (2000) and De Brecht and Saiki (2006) and also yields an approach to the motion detection dynamics. In Sect. 3.1, we test the model using both synthetic and 
natural-scene image sequences, focusing on a number of issues. First, we examine whether the processing latencies in our model are biologically plausible (also considered to some extent by De Brecht and Saiki (2006)). At this point, we do not perform direct comparisons to electrophysiological experiments; we rather focus on the time taken by the system to reach the stable state once an object moves or suddenly appears (De Brecht and Saiki 2006; Yantis 1993). We also address the motion salience asymmetry phenomenon (experimentally described by Royden et al. (2001)) with our model. Finally, we also extend previous approaches (e.g., De Brecht and Saiki (2006)) by making predictions of the attended locations by the human eye during dynamic scene viewing, and compare the model behavior with and without motion salience detectors. In addition, there are strong considerations in relation to the Lyapunov stability in our model (Wilson 2004); and in Sect. 3.2 we discuss in more detail mathematical considerations of the model.

We evaluate the performance of the visual attention model mainly in cluttered scenes, including synthetic scenes that have been used in previous simulations. In addition, we have included preliminary consideration of complex motion patterns such as the EC Funded CAVIAR project (2011) database scenarios (the databases used to evaluate our approach are described below). The frames were shown to the network at a frequency of $25 \mathrm{frames} / \mathrm{s}$, i.e., one frame every $40 \mathrm{~ms}$. We found that this time was enough for the system to integrate the stimulus.

We next describe the data sets used and the experimental setup in this work. In general, we used the same procedure for preliminarily evaluation of both datasets. According to the holders' description, these data sets were recorded in fairly different conditions (lighting, camera positions, etc.), which introduce some good variability on important features for saliency computation. On the other hand, there is no variability regarding the dimensions of the analyzed frames.

\subsection{Preliminary empirical evaluation of the dynamical neural network model}

\subsubsection{Dynamical scene data sets}

Video Sequence Database (ICPR Workshop, Cambridge, United Kingdom, 2011) All these sequences were tested under three different lighting conditions described in this database. The sequences were recorded with a head mounted camera at 25 frames/s, each frame with approximate dimensions: $768 \times 584$ pixels. A smaller downscaled losslesscompressed version of the database to $384 \times 292$ pixels was used in order to keep the processing times and computational load under control.
EC Funded CAVIAR project database test case scenarios This database contains a number of video clips from different scenarios of interest. These include people walking, entering and exiting shops, among others. There are two main sections of video clips. The first one was recorded using a wide angle camera lens at 25 frames/s and compressed using MPEG2 with fixed resolution of $384 \times 288$ pixels. The second set of data also used a wide angle lens along and across the hallway in a shopping mall. For each sequence, there were two synchronized videos; one with a view across the hallway and another with a view along the hallway. This set of sequences are longer (approximately 1,500 frames), containing more moving individuals, groups and complex motion patterns depending on the view. There were no further preprocessing steps for the images of this database.

\subsubsection{Results on synthetic and natural, cluttered scenes}

Saliency processing latencies Results of our main experiments are summarized in Figs. 3, 4, 5, 6, 7, 8, 9 (for synthetic and natural-scene image sequences). We included some random noise to compute saliency on synthetic scenes in order to confirm the properties of our model in comparison to the Itti et al.'s (1998) model.

Depending on the movement traces, the target activity rises above the distractors' activity within $100 \mathrm{~ms}$ (the time in which the integration of the system is performed to produce a steady-state response). When one single object (the most salient or another) sequentially moves, the transitory response of the system exhibits several overshoots that cause the stimulus to appear temporally more salient than others. Approximately $100 \mathrm{~ms}$ later, populations' activity reaches the steady state, illustrating the fact that when the stimulus stops moving, the visual system activity decays (De Brecht and Saiki 2006; Desimone and Duncan 1995; Desimone and Ungerleider 1989). Under the assumptions from De Brecht and Saiki (2006), typical pop-out search reaction time is around 500-600 ms (200-300 ms for the simple reaction time); taking approximately $100 \mathrm{~ms}$ to compute saliency seems to be reasonable (Yantis 1993).

Conversely, when motion occurs for one object other than the most salient of the scene, as in Fig. 4, the priority of the moving object seems to decay faster than in other cases; however, as its activity stabilizes in about $100 \mathrm{~ms}$ (like in the other cases), the most salient object becomes more important provided that its primary visual features encode higher saliency levels, i.e., its level of activity rises above the distractors'. Under these assumptions it is an expected effect from the model that relevant non-salient objects elicit weaker neural activity and that the increased neural activity induced by the most salient objects in static conditions produces its faster decay. Additional remarks with regards to the system's 
(a)

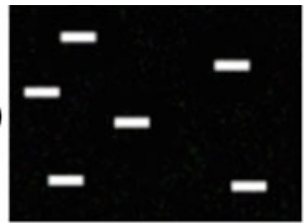

(b)

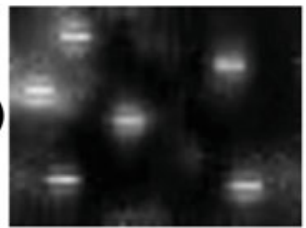

(1)
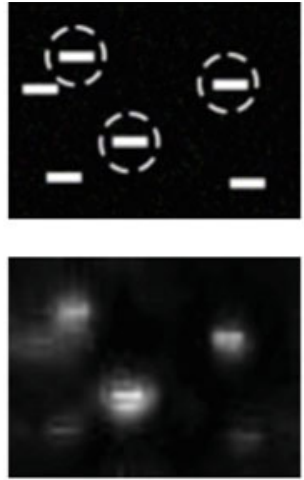

(2)
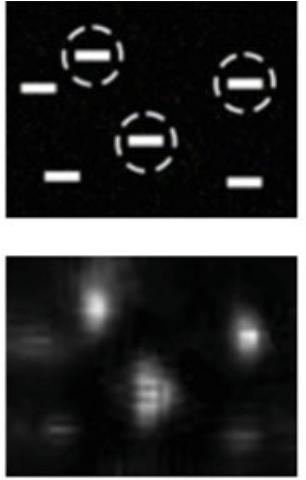

(3)

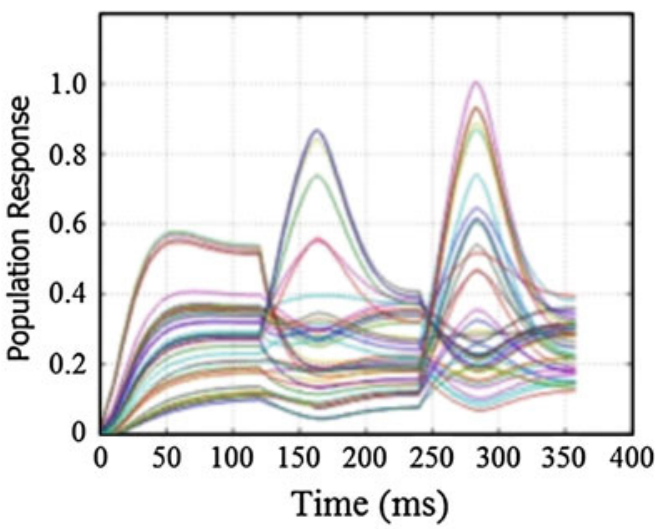

(4)
Fig. 3 a Representative movie frames (adding 20\% random color noise): (1) moving objects (white bars, frame 1) marked using white circles; $(2,3)$ objects (subsequent representative frames) performing translations. b Population responses: (1) initial saliency map activity before the moves occur; $(2,3)$ saliency map's transient response (over- shoots) when the already salient stimuli move; (4) time response representing the saliency map activity over $440 \mathrm{~ms}$ (note that $50 \%$ of the objects move at the same time and perform two movements on this sequence occurring approximately after 120 and $250 \mathrm{~ms}$ ). Normalized values on the $y$ axis
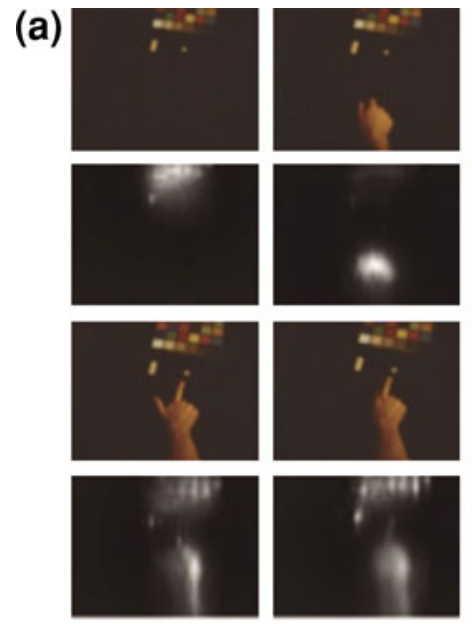
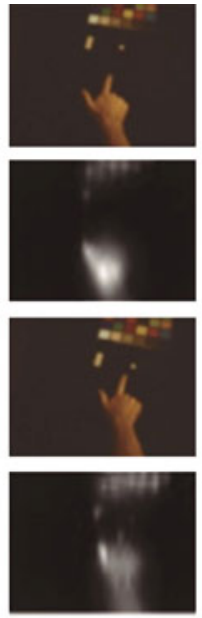

Fig. 4 a (Top rows) Representative movie frames. (Bottom rows) Saliency map's transient responses (overshoots) when stimuli move (multiple objects move at the same time and perform multiple move-

analysis without the motion saliency dynamics will be given later in the present section.

Saliency asymmetries A classical result in psychophysics is the existence of saliency asymmetries (e.g., Gao and Vasconcelos (2007)). It has been of great interest to investigate whether or not the so-called asymmetry phenomenon exists between moving and stationary targets (Wolfe 2001; Horowitz et al. 2007). A visual asymmetry refers to the situation in which it is easier to find a target of type A among distractors of type $\mathrm{B}$ than it is to find a target of type $\mathrm{B}$ among distractors of type A, see Figs. 3 and 5 (Wolfe 2001; Royden et al. 2001). As biological evidence points out, the reaction

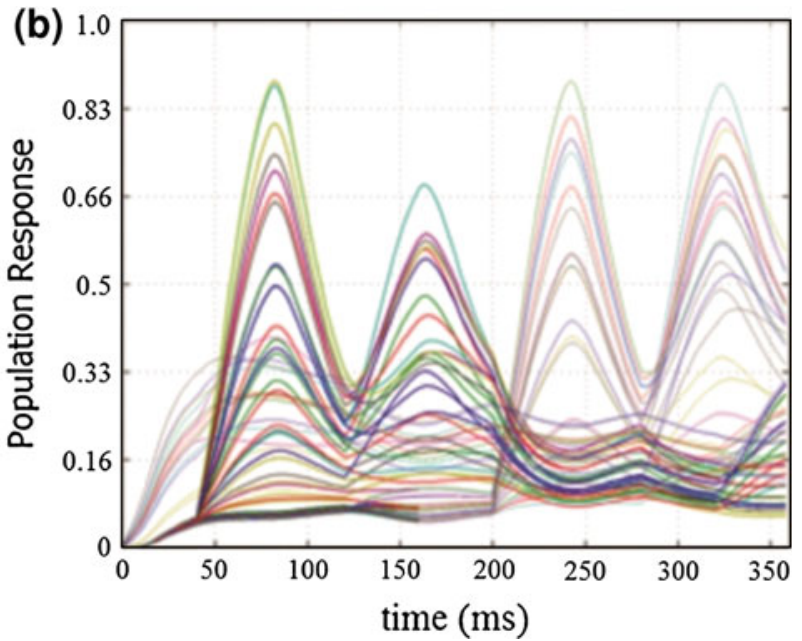

ments on this sequence: note that the camera moves and the neural responses increase for appearing objects). b Neural population response representing the saliency map activity. Normalized values on the $y$ axis

times increase dramatically when the subjects look for a stationary target among moving distractors. Reaction time also shows some dependence on the number of moving distractors and on the type of motion performed by such moving distractors (Itti et al. 1998).

Consequently, consistent with psychophysics, we evaluated our model with a series of video sequences as typically used in various experimental studies of visual motion asymmetries (Wolfe 2001; Royden et al. 2001). We ran the model over ten different movie sequences in order to make evident asymmetry in visual motion search. These movie sequences were distributed on three main motion patterns: linear, random linear, and Brownian. The results of this part of our 

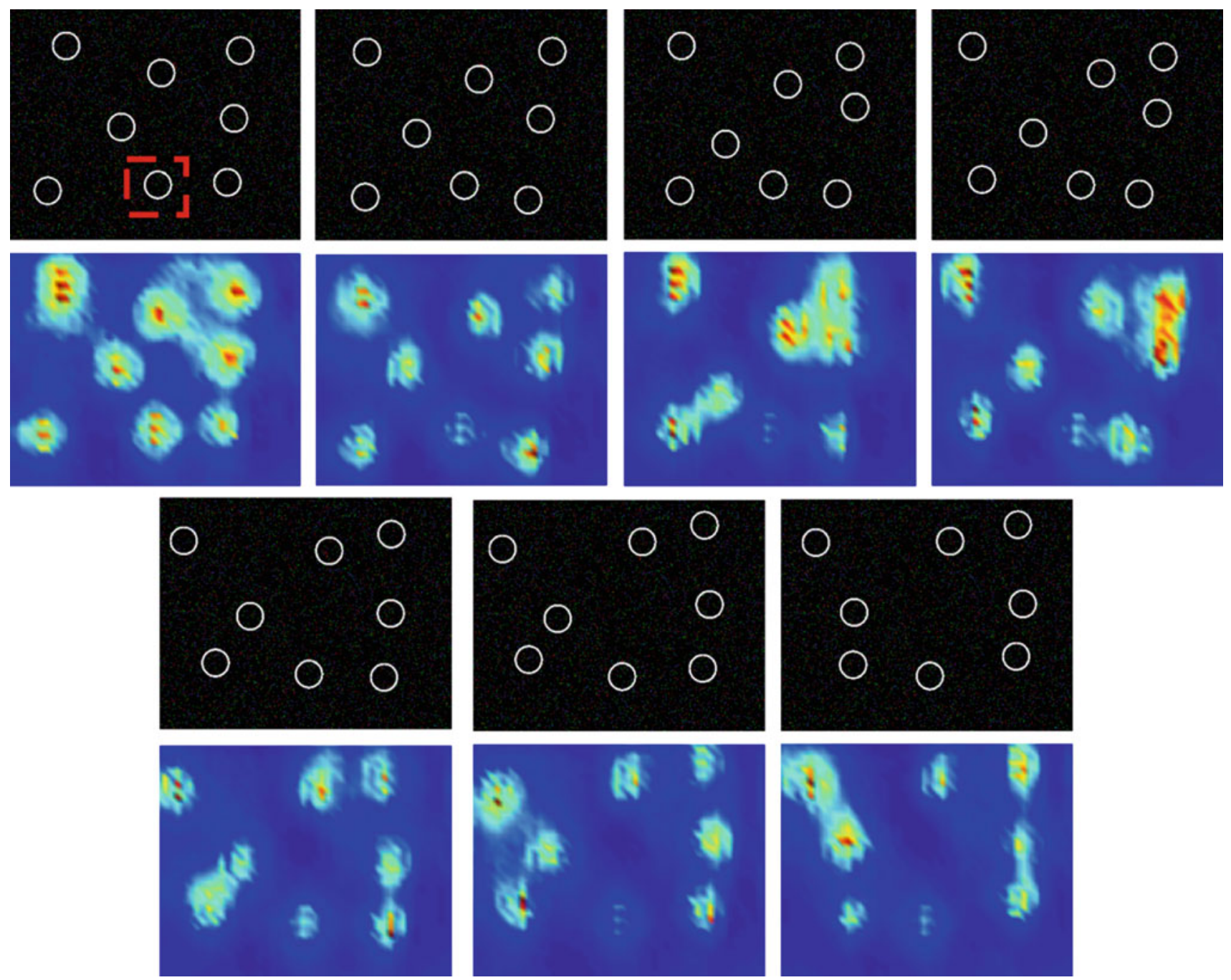

Fig. 5 Motion visual search asymmetries (the object marked in red remains static during the course of the movie sequence): (top rows) representative movie frames; (bottom rows) neural network transient responses (overshoots). (Colors are necessary here to enable the reader to interpret the figure)

work showed the saliency model reproduces motion search asymmetries for feature presence vs. feature absence-that have been previously reproduced on static scene analysisbased models (Gao and Vasconcelos 2007; Itti et al. 1998). Figure 5 illustrates that the approach described in this paper reproduces visual motion asymmetry properties: The objects that elicit the higher neural activity are those that are moving rather than the objects that remain stationary.

The network's topology and connectivity support to some extent the neurobiological basis of the visual motion search asymmetry phenomenon. One possibility is that the motion among distractors (especially for Brownian motion pattern) makes the system highly responsive to changes that cannot be easily "clustered" and therefore relegated as a common feature in order to allow the static object to be more salient than others. As other researchers have suggested, asymmetries exist with other basic visual features as well (Wolfe 2001; Nagy and Cone 1996; Treisman and Gormican 1988).
For instance, it is likely that visual motion information in the case of Brownian motion, Fig. 5, elicits stronger neural responses given the "novelty" presented by frame-to-frame motion traces (Royden et al. 2001). Consequently, the results illustrated in Fig. 5, point out that perceptual organization must be built in the global context of the movement of other items. In other words, the visual system is sensitive to movement coherence; items moving coherently are more easily grouped (Matsuno and Tomonaga 2006). For all the experiments carried out in this work, the model presented low neural responses for non-moving objects. Another important fact is that the collaborative and competitive interactions among the neurons of the saliency map (the adaptation strength among neurons), facilitate nearby elements to rise up with respect to the others.

Motion detection and motion saliency Results clearly show the main effect of the motion detection dynamical equations 

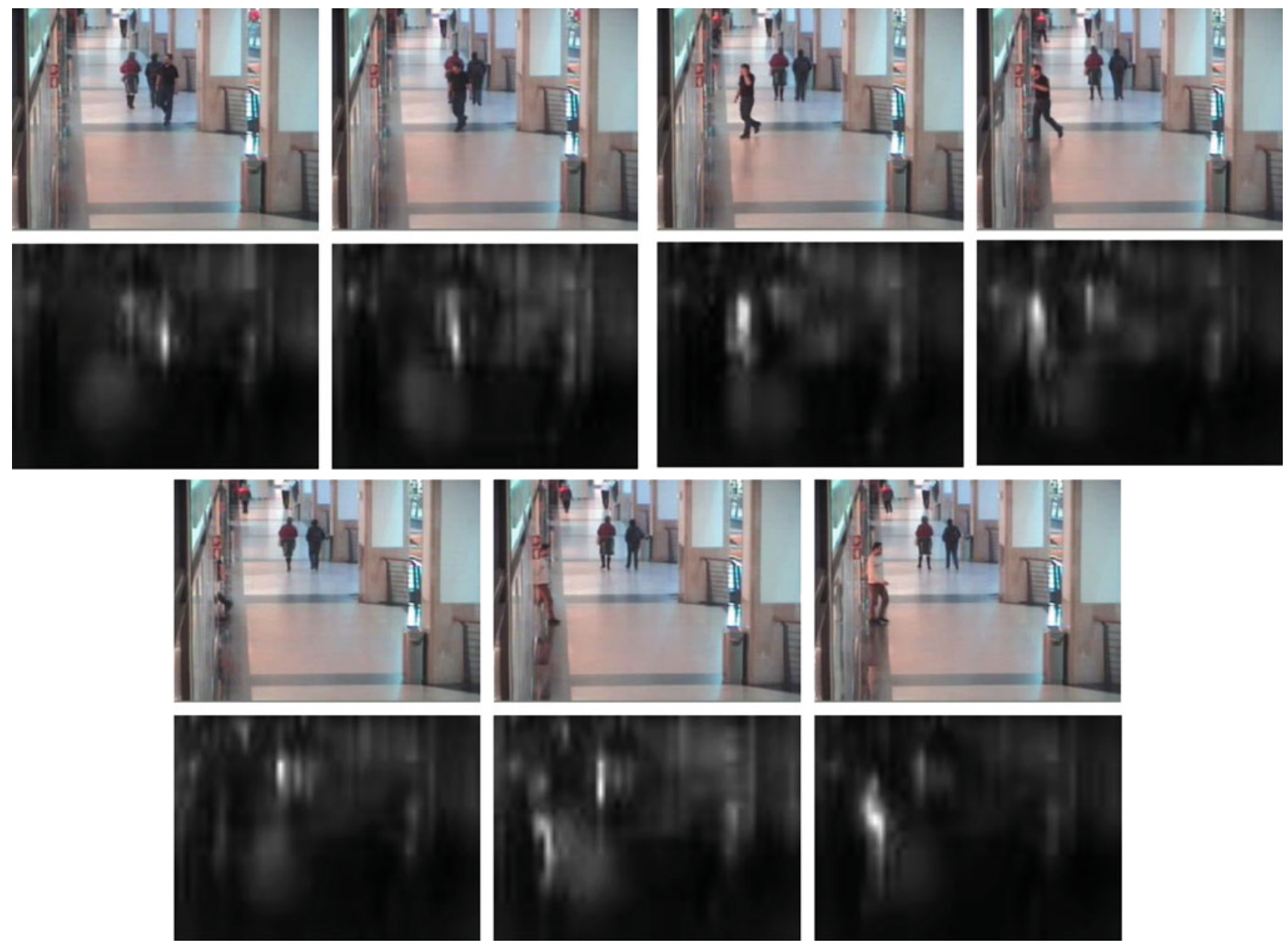

Fig. 6 (Top rows) representative movie frames. (Bottom rows) Saliency map's transient responses (overshoots) when stimuli move (multiple objects move at the same time and perform multiple movements on this sequence)

and their integration to the dynamical saliency map represented by arrays of neural populations. As a general statement, while the objects move over the entire dynamical scene, attention is attracted by the moving targets, and their other features (such as intensity, orientation and color) modulate their instantaneous overall priority (if we consider each frame as a static image). When the moving target is the most salient of the scene under static conditions, as in Figs. 6 and 8, the observed effect over the other objects (distractors) is an evident decrease of their relative activity. However, these objects are not eliminated from the central dynamical representation as this is an expected effect derived from the synaptic depression: As the moving target-related activity increases, its self-excitatory connections will become weaker; the same effect is expected for inhibitory connections to surrounding neuron populations (this accounts for automatic dynamical normalization of synaptic connections). It is important to note that the neural responses in Figs. 3 and 4 are the overshoots (transitory responses) of the system to moving stimuli.
In all the analyzed cases (the available sequences from the two video databases), the network elicited strong responses for simple motion patterns (translations) in the dynamical scenes. Complex motion patterns such as contractions or reductions, rotations or combinations of these kinds of movements did not elicit strong transients in the network, since those require more sophisticated visual elaborations and therefore modeling of neuronal structures of higher level (Tsotsos 2001).

To measure the statistical difference between moving and non-moving objects' saliency (only for synthetic images), we computed several KS-tests for the main saliency cases stated in 20 different attempts (single or multiple object movements) which are indirectly considered in the databases: (1) When the most salient object of the scene performs a single move; (2) when one object other than the most salient one makes a single move; (3) when the most salient or other object makes multiple moves; and (4) when two or more objects of the scene make a single or multiple moves. The main results of our experiments are depicted in Fig. 7. 

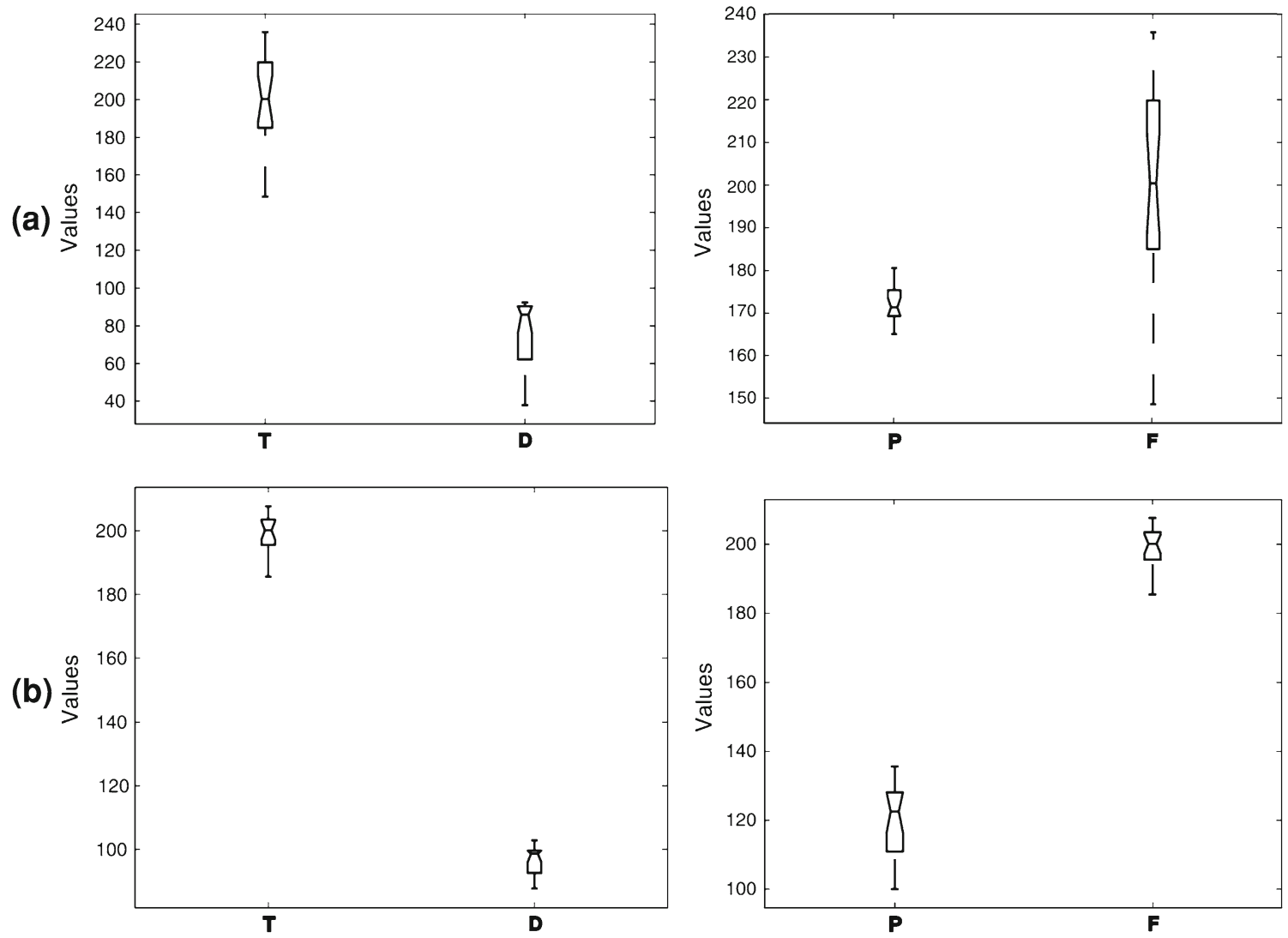

Fig. 7 (Left column) Objects' mean neural activity for target $(T)$ and distractor(s) $(D)$ during the network's transitory response. (Right column) Objects' mean neural activity before $(P)$ and after $(F)$ performing

As expected, there is statistical significance related to the comparison between the mean saliency of moving and nonmoving objects $\left(P<10^{-9}\right)$ during the transient response, even when the moving object is not the most salient object of the initial static scene $\left(P<10^{-9}\right)$. Moreover, it was expected that when comparing the samples of the two groups (when the moving object was or was not the most salient), there were statistical differences especially between the distractors' activity $\left(P<10^{-5}\right)$ : As the static primary visual features (intensity, color, and orientation) compute the overall priority of the input stimuli, higher mean saliency values are expected for the distractors in the second case.

Comparison of model with and without motion detector salience De Brecht and Saiki (2006) described a computational neural network model that integrates time responses; it appeared to be highly suitable to explore sudden-onset as well as moving stimuli. However, their approach would definitely fail when the moving object is other than the most salient: the model's dynamics itself cannot force an adequate response when the moving target is a non-salient object in one given instantaneous static representation, the reason is that there is no dynamic biasing effect in the dynamical equations in motion (transitory response). a The moving object was the most salient of the scene. $\mathbf{b}$ The moving object was not the most salient of the scene

order to treat favorably the moving targets. In other words, the overshoot produced by the activity of the neurons that remained silent in the scene before motion, is not enough to produce the transitory moving target stimulus to rise above the non-moving distractors in the overall cells' population activity; this leads to the necessity of implementing motion detection dynamical equations. For reference, Fig. 8 illustrates our model's dynamics in absence of the motion detection equations. Note that the changes along the frames elicit some activity in the network, as the neurons of the map are activated by the presence of the stimuli; however, the model fails to produce transient responses in which the activity of the moving object(s) rises above the distraction objects as can be noticed from the encircled regions in Fig. 8. Note that, the model computes static salience but the attention is not attracted by moving stimuli. Presumably, this effect would be also familiar to the De Brecht and Saiki (2006) dynamical model.

As a complement to the formerly reported results, additional KS-tests were performed in order to assess the difference between the neuronal activation levels of the network with dynamical motion detectors and without dynamical motion detectors. For such purpose, 20 consecutive movie 


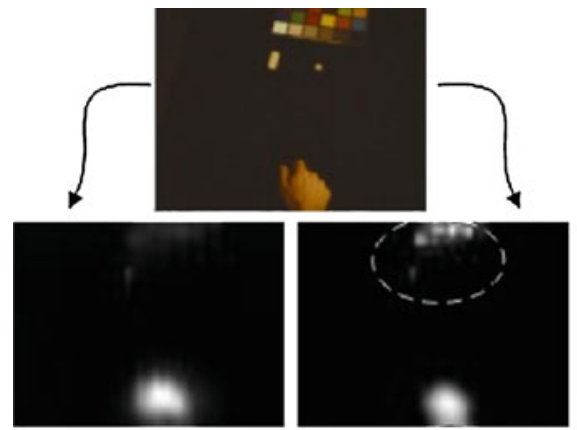

$\left({ }^{*}\right)$

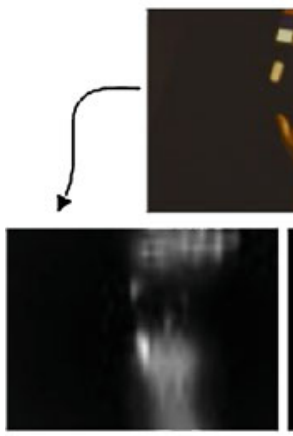

$(*)$

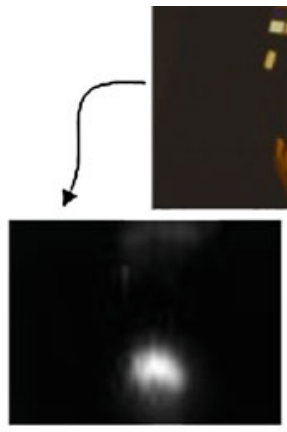

$\left({ }^{*}\right)$

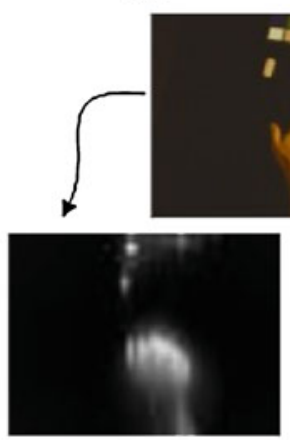

$(*)$

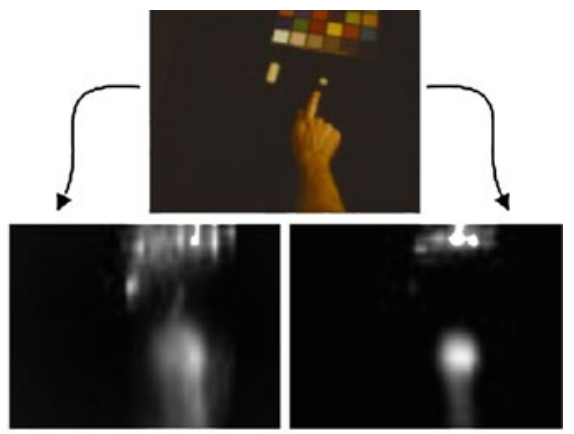

$(*)$

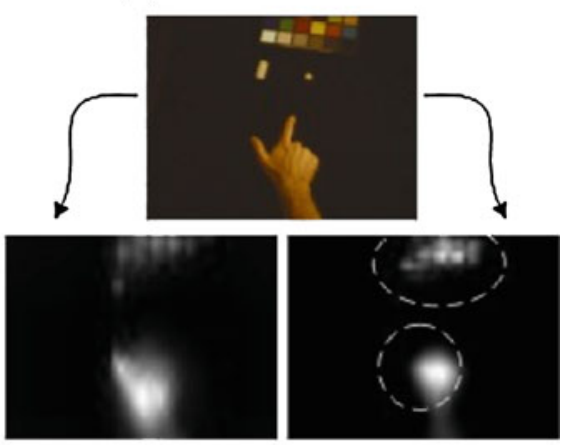

$(*)$
Fig. 8 Visual comparison between populations' responses with and without dynamical motion detectors. Top rows example crucial movie frames, in which the hand is moving as shown across the frames, and the other object is still. Bottom rows Saliency maps' transient responses

frames were taken from 20 randomly selected movies from the databases previously described (in this case, a total of 200 frames were analyzed). All comparisons were made for the difference in activation levels elicited by moving targets versus static distractors during the peak of the transitory response of the network. From this analysis, one should expect the motion detection dynamics to lead to a higher moving target-to-distractors difference during the transitory response of the network, thereby properly encoding the dynamically changing stimuli in time. On the other hand, in the absence of such dynamics, one should observe that the activity elicited by moving targets is as high as the activity elicited by static distractors during the entire time course of the network's activity.

The results of the above mentioned statistical procedure are depicted in Fig. 9. The difference in activity levels between moving targets and distractors showed increased statistical significance $\left(P<10^{-50}\right.$ ) between the network's activity levels with dynamical motion detectors and the network's activity levels without dynamical motion detectors. As expected, such difference was larger in the former case (with 95\% confidence interval mean values $104.7346 \pm$ 2.0650 versus $57.2649 \pm 2.0650$, respectively). In addition, in general, we observed two effects directly related to the dynamical motion detectors: on one hand, higher saliency with model that either includes motion detectors (right side; marked by asterisk) or that does not include motion detectors (left side). Note that, the regions encircled by the dashed lines highlight the regions that the model without motion detection does not process properly

values were elicited by moving targets during the network's transitory response in presence of motion detectors compared to those elicited in absence of them $\left(P<10^{-20}\right.$, not showed in the figure); on the other hand, lower saliency values were elicited by static distractors during the network's transitory response in presence of motion detectors compared to those elicited in the absence of them $\left(P<10^{-16}\right.$, not showed in the figure). These facts, taken together, emphasize the main role of the motion detection mechanism in the network's dynamics (see also Figs. 10, 11). To this end, the network's temporal response and the stability of its dynamics are highly sensitive to dynamical motion detection and to the free parameters involved in such mechanism (although both the system with motion detection dynamics and its reduced form are Lyapunov asymptotically stable).

\subsection{Mathematical considerations}

The purpose of this section is to provide a mathematical general overview of the dynamics of our neural network. We will consider two cases: for the first one, we will consider the effect of the synaptic depression for a network of one population; and for the second one, we will go through a more complex network of two populations as it appears in our work. 

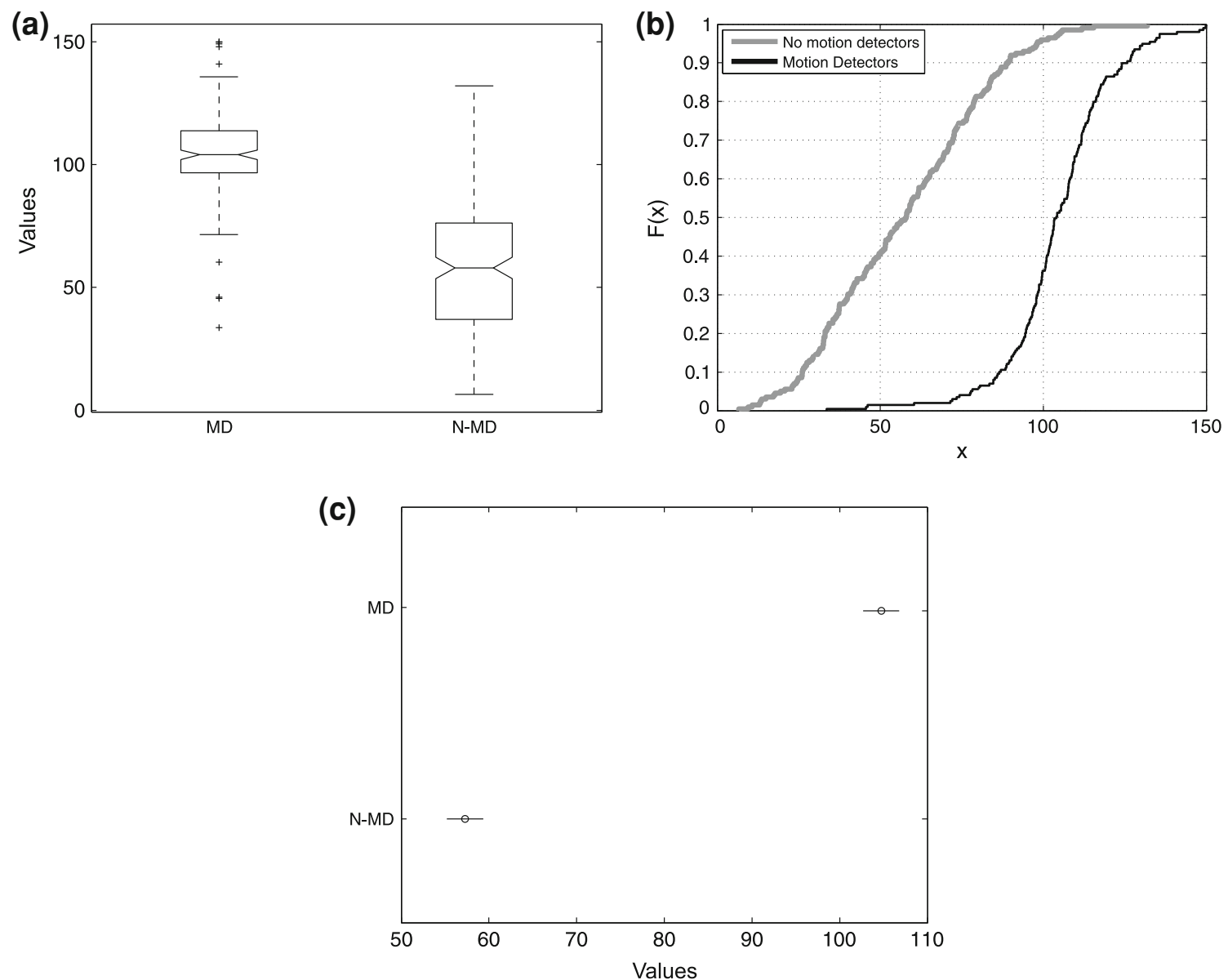

Fig. 9 Statistical results regarding the difference in activity levels between moving targets and static distractors. The comparison are established between the network's activity levels with dynamical motion detectors (MD) and the network's activity levels in the absence of dynamical motion detectors (NM): a box plots; b cumulative distribution functions (CDF); and c comparison plot for the mean values of the distributions. For all purposes, $F(x)$ is the cumulate distribution of the values of the random sample, and $x$ runs over the distribution values. The multiple comparison test plot $\mathbf{c}$ illustrate the mean of each set/group (corresponding to either MD or N-MD condition) and the standard error bars around the mean. Furthermore, the $95 \%$ confidence interval (CI) for the difference between the means was (43.3311-51.6082)
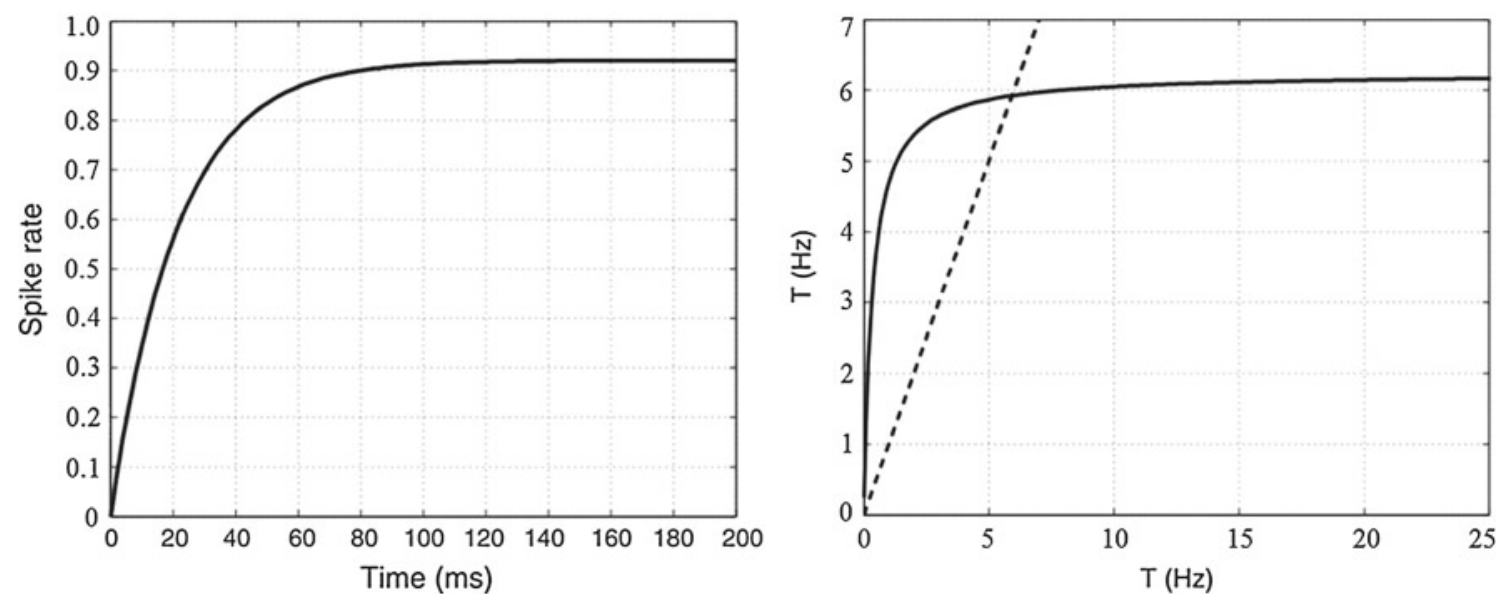

Fig. 10 Solution of the system of equations for the network of one population: (left) Numeric solution of the dynamic equations. Normalized values on the $y$ axis. (Right) graphical solution of the fixed-point equation 
First of all, let's consider the simplest case of our network, i.e., a neural network of only one population with the Naka-Rushton term embedded into the mean field equations with synaptic depression. Consequently, our system reduces to:

$\tau_{1} \frac{\mathrm{d} T}{\mathrm{~d} t}=-T+f\left(E_{T_{k}}+x_{1} T\right)$,

$\frac{\mathrm{d} x_{1}}{\mathrm{~d} t}=-x_{1} U_{\mathrm{SE}}^{L} T+\frac{1-x_{1}}{\tau_{2}}$,

For the fixed point, we obtain the following resulting equations:

$T-f\left(E_{T_{k}}+x_{1} T\right)=0$,

$-x_{1} U_{\mathrm{SE}}^{L} T+\frac{1-x_{1}}{\tau_{2}}=0$,

By standard substitution we obtain:

$T=f\left(E_{T_{k}}+\frac{1}{U_{\mathrm{SE}}^{L} \tau_{2} T+1} T\right)$,

Using the standard model parameters chosen in this work, i.e., $C=40.0 ; \quad \sigma=120.0 ; \quad \tau_{2}=10.0 ; \quad U_{\mathrm{SE}}^{L}=1.2$, we obtained the graphical results in Fig. 10.

Linearization of Eqs. (28-29) around any steady states can be obtained as follows:

$\frac{\mathrm{d} \underline{f}}{\mathrm{~d} \underline{x}}=\left[\begin{array}{ll}\frac{\partial f_{1}}{\partial x_{1}} & \frac{\partial f_{1}}{\partial x_{2}} \\ \frac{\partial f_{2}}{\partial x_{1}} & \frac{\partial f_{2}}{\partial x_{2}}\end{array}\right]=\left[\begin{array}{lc}-1-S^{\prime} x_{1} & S^{\prime} T \\ -x_{1} U_{\mathrm{SE}}^{L} & -U_{\mathrm{SE}}^{L} T-\frac{1}{\tau_{2}}\end{array}\right]$,

where $S^{\prime}$ is the derivative of the Naka-Rushton function evaluated at the steady state. The eigenvalues of Eq. (31) are:

$$
\begin{array}{r}
\lambda_{1}=-\frac{\sqrt{L}-\tau_{2} S^{\prime} x_{1}^{*}+U_{\mathrm{SE}}^{L} \tau_{2} T^{*}+\tau_{2}+1}{2 \tau_{2}} ; \\
\lambda_{2}=\frac{\sqrt{L}+\tau_{2} S^{\prime} x_{1}^{*}-U_{\mathrm{SE}}^{L} \tau_{2} T^{*}-\tau_{2}-1}{2 \tau_{2}}
\end{array}
$$

where

$$
\begin{aligned}
L= & S^{\prime 2} \tau_{2}^{2} x_{1}^{* 2}-2 S^{\prime} \tau_{2} x_{1}^{*}\left(U_{\mathrm{SE}}^{L} \tau_{2} T^{*}+\tau_{2}-1\right) \\
& +U_{\mathrm{SE}}^{L 2} \tau_{2}^{2} T^{* 2}+2 U_{\mathrm{SE}}^{L} \tau_{2} T^{*}\left(1-\tau_{2}\right)+\tau_{2}^{2}-2 \tau_{2}+1
\end{aligned}
$$

and $T^{*}$ and $x_{1}^{*}$ are the values of $T$ and $x_{1}$ at the fixed point.

As the response of $T$ increases, $x_{1}$ response must decrease in order for the input to counterbalance the activity elicited by $T$. In the absence of $x_{1}$ it is evident that the unbounded growing of $T$ will occur until saturation. On the other hand, for eigenvalues with negative real part, the system will be Lyapunov asymptotically stable. It has become obvious that the stability of the system depends on the constants of the model, for which we have recommended to use certain ranges given the extensive experimentation we have performed in our work. Like in (Tsodyks et al. 1998), our formulation allows for an analysis of the behavior of the network with any pattern of connections, internal dynamical interactions and external inputs. All these factors have been taken into account for the current dynamical model.

Conversely, for the second case, we are going to consider two competing neural populations with the synaptic depression effects for both of them. In this case, the model is simplified to:

$\tau_{1} \frac{\mathrm{d} T}{\mathrm{~d} t}=-T+f\left(E_{T_{k}}-r N D+x_{1} T\right)$,

$\tau_{1} \frac{\mathrm{d} D}{\mathrm{~d} t}=-D+f\left(E_{D_{k}}-r(N-1) D-r T+x_{2} D\right)$,

$\frac{\mathrm{d} x_{1}}{\mathrm{~d} t}=-x_{1} U_{\mathrm{SE}}^{L} T+\frac{1-x_{1}}{\tau_{2}}$,

$\frac{\mathrm{d} x_{2}}{\mathrm{~d} t}=-x_{2} U_{\mathrm{SE}}^{L} D+\frac{1-x_{2}}{\tau_{2}}$,

Linearization of Eqs. (32-35) around any steady states is obtained as follows:

$\underline{\mathrm{d} \underline{f}}=\left[\begin{array}{llll}S^{\prime} x_{1}-1 & -r N S^{\prime} & T S^{\prime} & 0 \\ -r S^{\prime} & -1+S^{\prime}\left[x_{2}-r(N-1)\right] & 0 & D S^{\prime} \\ -x_{1} U_{\mathrm{SE}}^{L} & 0 & -U_{\mathrm{SE}}^{L} T-\frac{1}{\tau_{2}} & 0 \\ 0 & -x_{2} U_{\mathrm{SE}}^{L} & 0 & -U_{\mathrm{SE}}^{L} D-\frac{1}{\tau_{2}}\end{array}\right]$,

where $S^{\prime}$ is the derivative of the Naka-Rushton function evaluated at the steady state. The determinant solution of Eq. (36) is:

$d=\frac{-\left[\tau_{2} U_{\mathrm{SE}}^{L} D(A-(L+1) B)+\left(A-\left(\lambda-S^{\prime} x_{2}+1\right) B\right)\left(\tau_{2} \lambda+1\right)\right]}{\tau_{2}^{2}}$,

where

$$
\begin{aligned}
& A=r^{2} N S^{2}\left(\tau_{2} \lambda+\tau_{2} U_{\mathrm{SE}}^{L} T+1\right)+r S^{\prime}(1-N) \\
& \times\left(\tau_{2} \lambda^{2}-\lambda\left(\tau_{2} S^{\prime} x_{1}-\tau_{2} U_{\mathrm{SE}}^{L} T-\tau_{2}-1\right)\right. \\
& \left.-S^{\prime} x_{1}+\tau_{2} U_{\mathrm{SE}}^{L} T+1\right) \\
& B=\tau_{2} \lambda^{2}-\lambda\left(\tau_{2} S^{\prime} x_{1}-\tau_{2} U_{\mathrm{SE}}^{L} T-\tau_{2}-1\right) \\
& -S^{\prime} x_{1}+\tau_{2} U_{\mathrm{SE}}^{L} T+1
\end{aligned}
$$

As can be noticed, when there are two or more interconnected populations the complexity of the system increases dramatically and the responses elicited by the network for given stimuli and free parameters become in some cases cumbersome to interpret. Given this fact, there are a wide variety of responses that can be expected for different parameters. For instance, for the example illustrated in Fig. 11 we chose the same parameters as in our lead model in order to keep the latencies we reproduced in our work. In Fig. 11 one important effect can be noticed: when there is competition among neural populations and it is simulated in the absence of synaptic depression, there will be over-competitive behavior for any given 

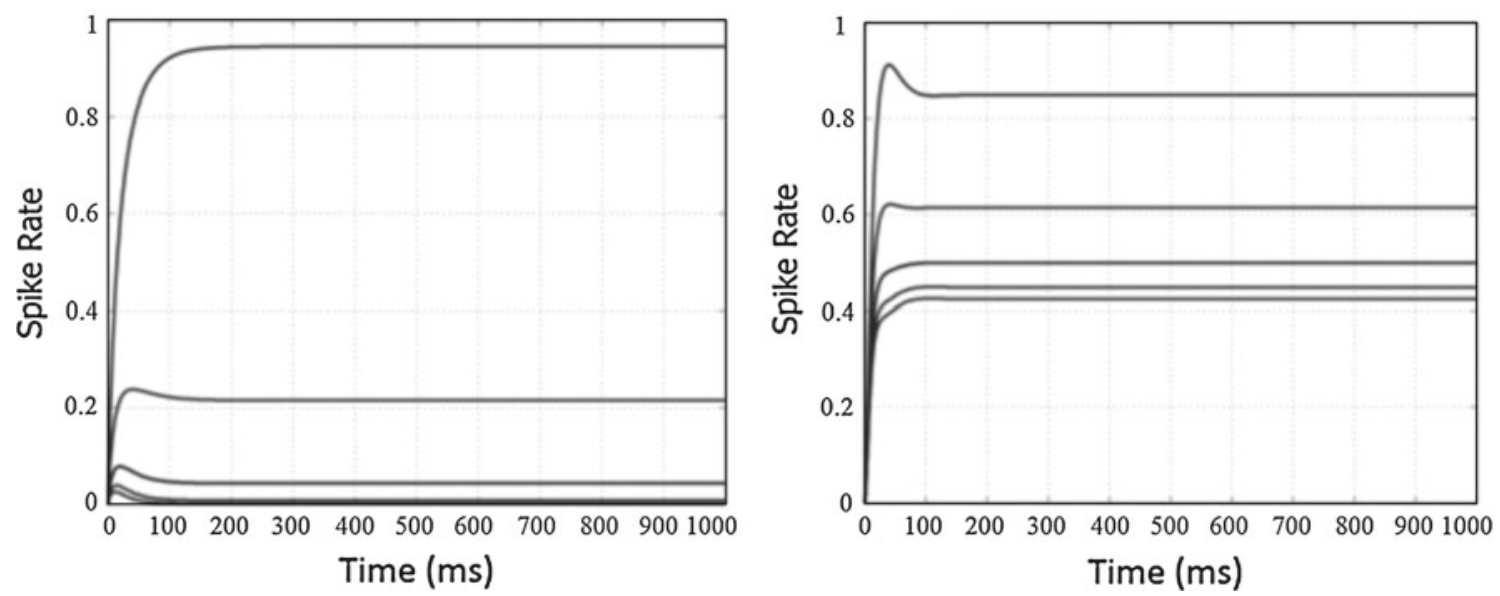

Fig. 11 Neural population responses: (left) in the absence of synaptic depression; (right) with synaptic depression. Normalized values on the $y$ axis

group of inputs even when the difference among them is not large; in consequence, the responses of such neural arrays will produce only one winner neuron. The equilibrium state of Eq. (32) in which $T$ neurons' firing rates at rate $T_{\text {eq }}$ will be asymptotically stable and the potential of such $T$ and $D$ neurons will depend on the effect of depression, a condition ensuring that all distractor responses will not drop to zero (given the responses of synaptic depression mechanism), which is undesirable and not neurobiologically reliable.

On the other hand, synaptic depression effects and inhibition constant $r$ must be balanced in order to allow a good trade-off between competition and the decrease of synaptic efficacy (especially for targets). Therefore, it is desirable that the activity elicited by the most salient object of the scene rises but at the same time distractors keep some active neural activity for the visual system to serially attend various locations by means of the so-called inhibition of return mechanism (as neurobiological evidence suggests). In addition, as the neural activity elicited by an object is not absolutely homogeneous for the correspondent representing neural array, an over-competitive model will select only one target represented by the activity of one neuron and therefore not the object itself represented by a local population activity as it occurs for objects in clutter. In light of these observations, this network, originally established as a WTA network (as it implemented the so-called winner-take-all competitive mechanism), will also implement neural decisions without the problem of over competition and will elicit strong transitory and steady state responses to moving objects with realistic processing latencies.

\section{Discussion}

We have demonstrated our model on a wide range of stimulus examples, including some synthetic examples
(Figs. 3, 5), motion asymmetry (Fig. 5), and real movies (Figs. 4, 6, 8). In addition to reproducing the main results of De Brecht and Saiki (see Figs. 3, 4), we have shown the relevance of including motion detectors into the dynamics of the neural network; indeed the network without them is insufficient to elicit an adequate response for certain moving stimuli (Fig. 8). The dynamical model for visual attention of De Brecht and Saiki (2006) focuses on some synthetic stimuli examples and does not consider the analysis of real, cluttered scenes. In addition, the feature extraction processes of De Brecht and Saiki are simplified, and do not include motion detectors. Their model considers sudden-onset stimuli, but they only briefly discuss issues regarding motion detection, which was our main focus here.

Furthermore, the literature consulted has addressed motion saliency processing either from a computational perspective (Park et al. 2002; Liu et al. 2007; Mahadevan and Vasconcelos 2010), or from a more closely biologically motivated perspective (De Brecht and Saiki 2006; Fix et al. 2010; Navalpakkam and Itti 2005, 2006; Walther and Koch 2006; Li 2002; Rapantzikos et al. 2007; Navalpakkam and Itti 2002; Peters et al. 2005); the latter was the path followed in our work.

The main contributions of our modeling work are twofold. First, the proposed model captures sudden-onset of less salient objects in dynamic scenes, compatible with suggestions in the experimental literature (e.g., Jonides and Yantis 1988). Our work is an attempt to describe how the visual system combines motion into a bottom-up salience strategy with realistic time responses and neural latencies as reported by perceptual data (Yantis 1993; Wilson 2004; Jonides and Yantis 1988), which suggest that when a subject carries out a visual inspection without looking after a feature singleton, then abrupt-onset singletons capture the attention (while other singletons do not, at least transitorily). Furthermore, sudden-onset stimuli grab visual attention and their priority over non-onset stimuli decays about $100 \mathrm{~ms}$ after onset. 
Fig. 12 Simplified framework of the model reported in this work

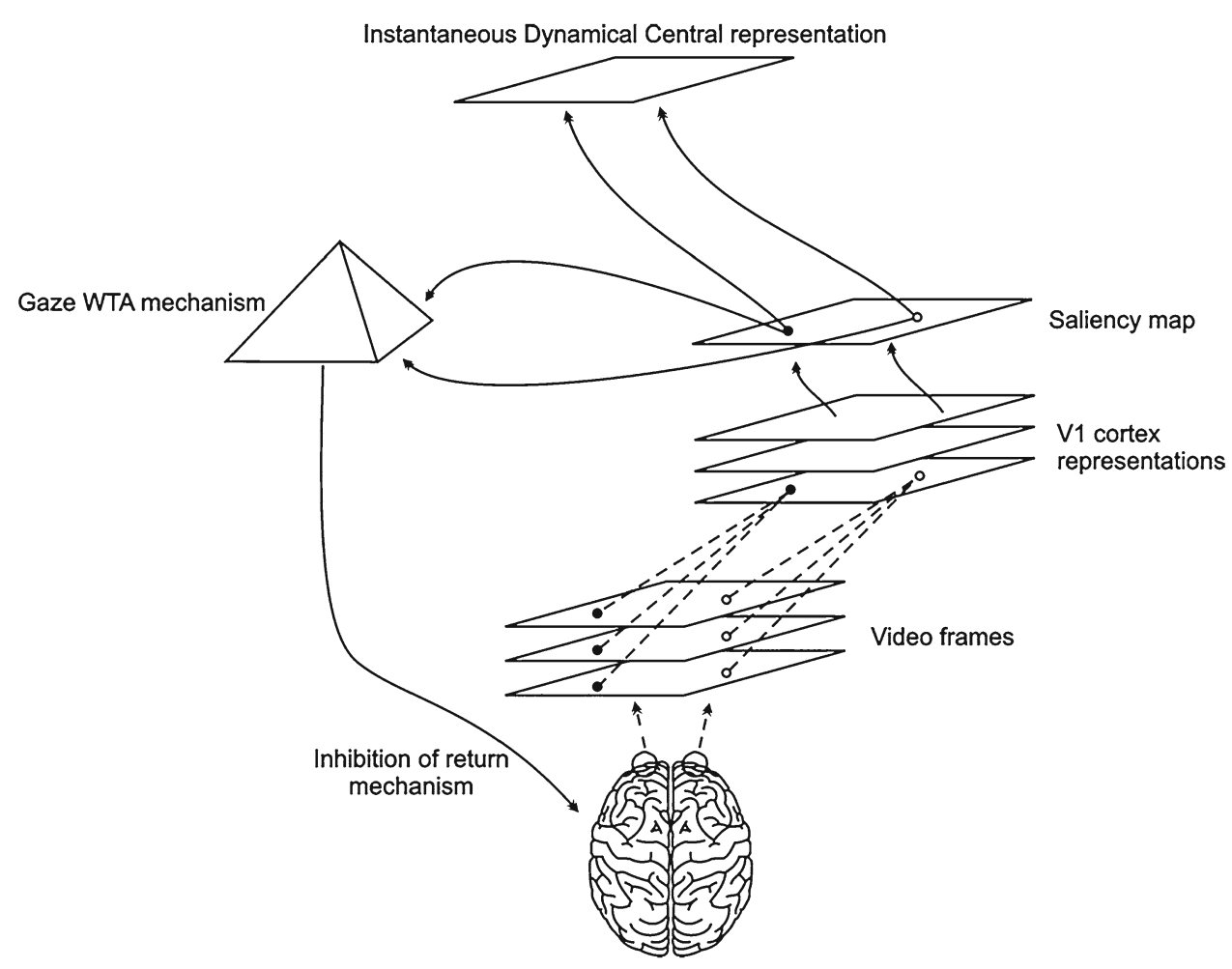

As shown by our results (with special regards to the motion detection dynamical equations), our model is a step toward visual modeling applied to dynamic environments and an attempt to reproduce the dynamics of visual neural populations during bottom-up visual search tasks in clutter.

Second, we showed the importance of keeping the synaptic depression of De Brecht and Saiki (2006) in our extended motion salience model: first, impeding unbounded growing of the moving target neural activity when it is the most salient; second, counterbalancing the effects of moving objects saliency with respect to non-moving objects, provided that in absence of depression these objects would disappear transitorily from the central representation; and third, helping non-salient objects to rise above others when they are under motion conditions or when they suddenly appear. Our model improves the results reported in Wilson (2004), regarding the neural mechanisms of visual processing of static scenes and those obtained by De Brecht and Saiki (2006) about motion and appearing stimuli. Therefore, our model is predicting that synaptic depression is useful not only for building a suitable saliency map for static conditions but also for dynamic scenes and inputs.

Our work relates to the dynamical saliency computation of attentional shifts in Itti (2004), but there they calculated saliency for the specific application of video compression. In this way, the dynamic nature of our model makes it potentially useful for saccade target selection or the so-called foveation mechanism modeling (Itti 2004; Pomplun 2007), given the increased interest to reproduce neural mechanisms of salient object tracking, which greatly simplifies computer analysis of dynamic scenes. The so-called virtual foveas (Itti 2004; Burt 1988) do not attempt to reproduce saccadic eye movements in a one-to-one eye implementation. Thereby, instead of modeling complex rapid ballistic eye movements (often erratic motions) that create visible artifacts, it is desirable to model it in a smooth and damped manner in order to track objects (Itti 2004). Incorporating such dynamics to our dynamical model will be a matter of future research efforts along with the consequent computer vision applications.

Figure 12 illustrates a simplified diagram of our model, including the potential involvement of area V1. Note, however, that we are not addressing V1 data in detail (such as extra classical receptive field effects and related perceptual phenomena), as has been done in V1 dynamical salience modeling approaches (e.g., Li (1999, 2002), and Zhaoping (2006)) and in statistical modeling [e.g., Coen-Cagli et al. (2012)]. Rather, we are including some of the properties as described by Itti (2004) and López et al. (2006). Further, this model can be expanded to modeling other visual processing centers such as area MT (responsible for the selectivity concerning the angle between local movement direction and spatial velocity gradient), MST and 7a (responsible for more complex motion patterns analysis). Top-down volitional control is necessary too in order to complete the overall attention scheme with realistic dynamics.

Our model only addresses bottom-up processes, and can be adapted and extended to models that integrate topdown processes. Different features contribute to perceptual 
saliency and their weighing can be influenced by top-down modulation (Deco and Rolls 2004; Fahy et al. 1993; Cauller 1995; Connor et al. 2004; Oliva et al. 2003; Peters et al. 2005; Serre et al. 2006; Mira et al. 2006; Sobel et al. 2009). There is experimental evidence concerning strong interactions among different visual modalities such as color and orientation for certain visual locations (Nothdurft 2000); these interactions are subject to top-down modulation and training. In this way, the attention process could select the necessary information to discriminate between the distractors and the target while both bottom-up and top-down processes are carried out to analyze the same scene: here the top-down process is related to previously acquired knowledge that biases the neural processing competition among the objects; and recognition could be performed by selecting the next eye movement that maximizes the information gain. The computational challenge, then, lies in the integration of bottom-up and top-down cues, such as to provide coherent control signals for the focus of attention, and in the interplay between attentional orientating and scene or object recognition (Itti and Koch (2001); see also Mira et al. (2006)). We consider two possibilities for achieving integration of bottom-up and top-down in our framework in the future. First, a modulation of the objects' initial representations at the earlier stages in our model could come from higher cortical centers which maintain the associations to the task at hand; this could be carried out via connections through an integrative neural area or nucleus like the superior colliculus. Second, that integration could be reached through interactions between the last computational stage in our model, the saliency map, and the higher cortical associative areas in charge of encoding the developing task.

Although the model proposed in this work relies exclusively on primary visual processing stages (bottom-up attention), Hamker (2004), Hamker (2006) has built a model that encompasses feature-based top-down modulation and bottom-up approaches as two independent and structurally different processes bearing a strong dynamical interaction. In its current stage, our model does not account for interaction with higher level visual processing centers or task-dependent attentional effects. Nonetheless, we have introduced a model architecture for which such extensions are possible, along with the possibility of reproducing cortical neuronal dynamics with realistic time responses.

Tsotsos (2001) and Tsotsos et al. (2005) proposed a feedforward pyramid-like network model for motion attention considering the following structures: area V1, area MT, area MST and area 7a (this is, to our knowledge, the most complete model in the literature regarding the analysis of simple and complex motion patterns). Their model accurately resembles the neurophysiological evidence informed by current knowledge of motion analysis in the primate cortex. Their model also explains how it is possible to integrate the information of certain processing centers and motion pattern attendance by selective tuning. Selective tuning in their model relies on a hierarchy of winner-take-all (WTA) processes with excitatory and inhibitory connections in a feed-forward pyramid-like network, adding the required feedback connections (including MST and 7a areas). Their model reproduces, at least qualitatively, some aspects of visual processing in human or primate vision. However, in their model, they do not address the motion analysis with realistic time responses. It would be interesting to integrate their feedforward/feedback approach, with more realistic dynamics of the kind we propose here.

Acknowledgments The authors of this work acknowledge the support of Ruben Coen-Cagli, Albert Einstein College of Medicine, NY, USA.

\section{References}

Abbott LF, Varela JA, Sen K, Nelson SB (1997) Synaptic depression and cortical gain control. Science 275:220-224

Bergen JR, Julesz B (1983) Parallel versus serial processing in rapid pattern discrimination. Nature 303:696-698

Bollman M, Hoischen R, Mertsching B (1997) In: Berlin et al. (ed) Integration of static and dynamic scene features guiding visual attention. Springer, Berlin, pp 483-490

Borst A (2000) Models of motion detection. Nature neuroscience 3:1168

Burt PJ (1988) Proceedings of the 9th international conference on attention mechanisms for vision in dynamic world. Patt Recog 1:977-987

Burt PJ, Adelson EH (1983) The Laplacian pyramid as a compact image code. IEEE Trans Commun 31:532-540

Cauller L (1995) Layer I of primary sensory neocortex: where top-down converges upon bottom-up. Behav Brain Res 71:163-170

Chen B (2005) Mathematical models of motion detection in the fly's visual cortex. Dissertation, Texas Tech University, Texas

Chen LQ, Xie X, Fan X, Ma WY, Zhang HJ, Zhou HQ (2003) A visual attention model for adapting images on small displays. Multimed Syst 9:1-12

Coen-Cagli R, Dayan P, Schwartz O (2012) Cortical surround interactions and perceptual salience via natural scene statistics. PLoS Comput Biol 8(3):e1002405

Connor CE, Egeth HE, Yantis S (2004) Visual attention: bottom-up versus top-down. Curr Biol 14:R850-R852

Conway BR (2001) Spatial structure of cone inputs to color cells in alert macaque primary visual cortex (V-1). J Neurosci 21:2768-2783

Conway BR (2009) Color vision, cones and color-coding in the cortex. The Neuroscientist 15:274-290

De Brecht M, Saiki J (2006) A neural network implementation of a saliency map model. Neural Networks 19:1467-1474

Deco G, Rolls ET (2004) A neurodyamical cortical model of visual attention and invariant object recognition. Vis Res 44:621-642

Desimone R, Duncan J (1995) Neural mechanisms of selective visual attention. Ann Rev Neurosci 18:193-222

Desimone R, Ungerleider LG (1989) Neural mechanisms of visual processing in monkeys. Elsevier, New York, pp 267-299

EC Funded CAVIAR project/IST 200137540 http://homepages.inf.ed. ac.uk/rbf/CAVIAR/. Accessed Jan 2011

Engel S, Zhang X, Wandell B (1997) Colour tuning in human visual cortex measured with functional magnetic resonance imaging. Nature 388:68-71

Fahy FL, Riches IP, Brown MW (1993) Neuronal activity related to visual recognition memory: long-term memory and the encoding 
of recency and familiarity information in the primate anterior and medial inferior and rhinal cortex. Exp Brain Res 96:457-472

Fix J, Rougier N, Alexandre F (2010) A dynamic neural field approach to the covert and overt deployment of spatial attention. Cogn Comput 3:279-293

Gao D, Vasconcelos N (2007) Bottom-up saliency is a discriminant process. Proceedings of the IEEE international conference on computer vision, Rio de Janeiro

Gonzalez Andino SL, de Peralta Grave (2012) Coding of saliency by ensemble bursting in the amygdala of primates. Front Behav Neurosci $6(38): 1-16$

Greenspan H, Belongie S, Goodman R, Perona P, Rakshit S, Anderson $\mathrm{CH}$ (1994) Overcomplete steerable pyramid filters and rotation invariance. Proc IEEE Comput Vis Patt Recog 1:222-228

Hamker FH (2004) A dynamic model of how feature cues guide spatial attention. Vis Res 44:501-521

Hamker FH (2006) Modeling feature-based attention as an active topdown inference process. BioSystems 86:91-99

Horowitz TS, Wolfe JM, DiMase JS, Klieger SB (2007) Visual search for type of motion is based on simple motion primitives. Perception 36:1624-1634

Ibbotson M (2001) Identification of mechanisms underlying motion detection in mammals. Springer, Berlin

Itti L (2004) Automatic foveation for video compression using a neurobiological model of visual attention. IEEE Trans Image Proc 13:1304-1318

Itti L, Koch C (2000) A saliency-based search mechanism for overt and covert shifts of visual attention. Vis Res 40:1489-1506

Itti L, Koch C (2001) Computational modeling of visual attention. Nat Rev Neurosci 2:194-203

Itti L, Koch C, Niebur E (1998) A model of saliency-based visual attention for rapid scene analysis. IEEE Trans Patt Anal Mach Intel 20:1254-1259

Jonides J, Yantis S (1988) Uniqueness of abrupt visual onset in capturing attention. Percept Psychophys 43:346-354

Kusunoki M, Gottlieb J, Goldberg ME (2000) The lateral intraparietal area as a salience map: the representation of abrupt onset, stimulus motion, and task relevance. Vis Res 40:1459-1468

Li Z (1999) Contextual influences in V1 as a basis for pop out and asymmetry in visual search. Proc Natl Acad Sci 96(18):10530-10535

Li Z (2002) A saliency map in primary visual cortex. Trend Cogn Sci 6:9-16

Liu T, Sun J, Zheng NN, Tang X, Shum HY (2007) Learning to detect a salient object. Proceedings of IEEE computer society conference on computer and vision pattern recognition, Providence

López MT, Fenández-Caballero A, Fernández MA, Mira J, Delgado AE (2006) Motion features to enhance scene segmentation in active visual attention. Patt Recog Lett 27:469-478

Mahadevan V, Vasconcelos N (2010) Spatiotemporal saliency in dynamic scenes. IEEE Trans Pattern Anal Mach Intell 32: 171-177

Matsuno T, Tomonaga M (2006) Visual search for moving and stationary items in chimpanzees (Pan troglodytes) and humans (Homo sapiens). Behav Brain Res 172:219-232

Maunsell JHR, Treue S (2006) Feature-based attention in visual cortex. Trends Neurosci 29:317-322

Meso AI, Zanker JM (2009) Speed encoding in correlation motion detectors as a consequence of spatial structure. Biological Cybern 100:361-370

Mira J, Delgado AE, Lopez MT, Fernandez-Caballero A, Fernandez MA (2006) A conceptual frame with two neural mechanisms to model selective visual attention processes. Neurocomputing 71:704-720

Mundhenk TN, Itti L (2005) Computational modeling and exploration of contour integration for visual saliency. Biol Cybern 93:188-212

Nagy AL, Cone SM (1996) Asymmetries in simple feature searches for color. Vis Res 36:2837-2847
Navalpakkam V, Itti L (2002) A goal oriented attention guidance model. Lect Notes Comput Sci 2525:453-461

Navalpakkam V, Itti L (2005) Modeling the influence of task on attention. Vision Res. 45:205-231

Navalpakkam V, Itti L (2006) Modeling the influence of task on attention. Proc IEEE Comput Soc Conf Comput Vis Pattern Recognit $1: 1-8$

Nothdurft H (2000) Salience from feature contrast: additivity across dimensions. Vis Res 40:1183-1201

Oliva A, Torralba A, Castelhano MS, Henderson JM (2003) Top-down control of visual attention in object detection. Proc Int Conf Image Proc 1:253-256

Park SJ, An KH, Lee M (2002) Saliency map model with adaptive masking based on independent component analysis. Neurocomputing 49:417-422

Peters RJ, Iyer A, Itti L, Koch C (2005) Components of bottom-up gaze allocation in natural images. Vis Res 45:2397-2416

Pointing Gestures: Video Sequence Database (ICPR Workshop, Cambridge, United Kingdom). http://www.cvmt.dk/. Accessed Jan 2011

Pomplun M (2007) Advancing area activation towards a general model of eye movements in visual search. In: Gray WD (ed) Integrated models of cognitive systems. Oxford University Press, New York, pp 120-131

Ramirez-Villegas JF, Ramirez-Moreno DF (2012) Color coding in the cortex: a modified approach to bottom-up visual attention. Biol Cybern. doi:10.1007/s00422-012-0522-6. Accessed on 28 Sept 2012

Rapantzikos K, Tsapatsoulis N, Avrithis Y, Kollias S (2007) Bottom-up spatiotemporal visual attention model for video analysis. Image Proc IET 1:237-248

Reynolds JH, Heeger DJ (2009) The normalization model of attention. Neuron 61:168-185

Royden CS, Wolfe JM, Klempen N (2001) Visual search asymmetries in motion and optic flow fields. Percept Psychophys 63:436-444

Santos A, Mier D, Kirsch P, Meyer-Lindenberg A (2011) Evidence for a general face salience signal in human amygdala. Neuroimage 54:3111-3116

Schrater PR, Knill DC, Simoncelli EP (2000) Mechanisms of visual motion detection. Nature Neuroscience 3:64-68

Sejnowski TJ, Koch C, Churchland PS (1988) Computational neuroscience. Science 241:1299-1306

Serre T, Wolf L, Bileschi S, Riesenhuber M, Poggio T (2006) Robust object recognition with cortex-like mechanisms. IEEE Trans Patt Anal Mach Intell 29:1-17

Shen K, Martin P (2007) Neuronal activity in superior colliculus signals both stimulus identity and saccade goals during visual conjunction search. J Vis 7(5):15: 1-13

Shormaker PA, O'Carroll DC, Straw AD (2001) Implementation of visual motion detection with contrast adaptation. Proc SPIE 4591:316-327

Sobel KV, Pickard MD, Acklin WT (2009) Using feature preview to investigate the roles of top-down and bottom-up processing in conjunction search. Acta Psychol 132:22-30

Thompson KG, Bichot NP (2005) A visual salience map in the primate frontal eye field. Prog Brain Res 147:251-262

T'so DY, Gilbert CD (1988) The organization of chromatic and spatial interactions in the primate striate cortex. J Neurosci 8:1712-1727

Torralba A, Castelhano MS, Oliva A, Henderson JM (2006) Contextual guidance of eye movements and attention in real-world scenes: the role of global features in object search. Psychol Rev 113:766-786

Trapp S, Schroll H, Hamker FH (2012). Open and closed loops: A computational approach to attention and consciousness. Adv Cogn Psychol 8(1):1-8. doi:10.2478/v10053-008-0096-y

Treisman A, Sykes M, Gelade G (1977) Selective attention stimulus integration. In: Dornic S (ed) Attention and performance VI. Lawrence Erlbaum Associates, New Jersey, pp 333-361 
Treisman AM, Gelade G (1980) A feature-integration theory of attention. Cogn Psychol 12:97-136

Treisman AM, Gormican S (1988) Feature analysis in early vision: evidence from search asymmetries. Psychol Rev 95:15-48

Tsodyks M, Pawelzik K, Markram H (1998) Neural networks with dynamic synapses. Neural Comput 10:821-835

Tsotsos JK (2001) Motion uniderstanding: task-directed attention and representation that link perception with action. Int J Comput Vis 45:265-280

Tsotsos JK, Liu Y, Matinez-Trujillo JC, Pomplun M, Simine E, Zhou $\mathrm{K}$ (2005) Attending to visual motion. Comput Vis Image Underst 100:3-40

Walther D, Koch C (2006) Modeling attention to salient proto-objects. Neural Networks 19:1395-1407

Wilson HR (2004) Spikes, decisions and actions: the dynamical foundations of neuroscience. Oxford University Press, New York
Wilson HR, Cowan JD (1973) A mathematical theory of the functional dynamics of cortical and thalamic nervous system. Kybernetik 13:55-80

Wolfe J, Butcher SJ, Lee C, Hyle M (2003) Changing your mind: on the contributions of top-down and bottom-up guidance in visual search for feature singletons. J Exp Psychol Hum Percept Perform 29:483502

Wolfe JM (2001) Asymmetries in visual search: an introduction. Percept Psychophys 63:381-389

Yantis S (1993) Stimulus-driven attentional capture and attentional control settings. J Exp Psychol Hum Percept Perform 19:676-681

Zhaoping L (2006) Theoretical understanding of the early visual processes by data compression and data selection. Network: Comput Neural Syst 17:301-334 